\title{
On the foundations of final coalgebra semantics: non-well-founded sets, partial orders, metric spaces
}

\author{
DANIELETURI $I^{\dagger}$ and JAN RUTTEN \\ ${ }^{+}$LFCS, University of Edinburgh, JCMB. The King's Buildings, \\ Edinhurgh EH9 3JZ, Scotland. \\ Emuil: dt@dcs.ed.ac.uk \\ ‡ CWI. P.O. BO. 94079 . \\ 1(190) GB Amsterdam. The Netherlands. \\ Email: Jan.Rutten@cwi.nl
}

Received 10 December 1996; retised 3 February 1998

This paper, a revised version of Rutten and Turi (1993), is part of a programme aiming at formulating a mathematical theory of structural operational semantics to complement the established theory of domains and denotational semantics to form a coherent whole (Turi 1996: Turi and Plotkin 1997). The programme is based on a suitable interplay between the induction principle, which pervades modern mathematics, and a dual, non-standard 'coinduction principle'. which underlies many of the recursive phenomena occurring in computer science.

The aim of the present survey is to show that the elementary categorical notion of a final coalgehra is a suitable foundation for such a coinduction principle. The properties of coalgebraic coinduction are studied both at an abstract categorical level and in some specific categories used in semantics, namely categories of non-well-founded sets, partial orders and metric spaces.

\section{Introduction}

The original stimulus for my own interest in the notion of a non-well-founded set came from a reading of the work of Robin Milner in connection with his development of a mathematical theory of concurrent processes. This topic in theoretical computer science is one of a number of such topics that are generating exciting new ideas and intuitions that are in need of suitable mathematical expression.'

Peter Aczel, Non-Well-Founded Sets

\footnotetext{
+ Research supported by the 'Stichting Informatica Onderzoek in Nederland' of the Dutch Organization for Scientific Research ('NWO') under grant 612-316-402, project 'Non-well-founded sets and semantics of programming languages'.
} 
In set theory, mathematical induction is based on the notion of a well-founded relation, that is, a relation $R$ such that, for every set $x$, there is no infinitely descending chain

$$
\ldots R x_{2} R x_{1} R x_{0}=x \text {. }
$$

The fact that standard mathematical constructions are inductive is mirrored by the common assumption that the axioms of set theory include the foundation axiom, which postulates that the set-membership relation ' $\epsilon$ ' is well-founded: for every set $x$, there exists no infinitely descending chain

$$
\ldots \in x_{2} \in x_{1} \in x_{0}=x .
$$

In Milner's Calculus of Communicating Systems (CCS), the behaviour of a non-deterministic program $P$ is given by the set

$$
\llbracket P \rrbracket=\left\{P \stackrel{a_{i}}{\longrightarrow} P_{i}\right\}
$$

of atomic transitions $P \stackrel{a_{i}}{\longrightarrow} P_{i}$ that the program can perform, producing an observable action $a_{i}$ and becoming $P_{i}$. The meaning of a program $P$ should abstract from the name of the programs involved in the transitions and focus on the actions that can be performed, together with the choices that can be made. It should, then, be the following 'coinductively' defined set:

$$
\left.\llbracket P \rrbracket^{@}=\left\{<a, \llbracket P^{\prime} \rrbracket^{@}\right\rangle \mid P \stackrel{a}{\longrightarrow} P^{\prime}\right\} .
$$

Now, in general, the transition relation is not well-founded, since, for instance, cyclic programs $P \stackrel{a}{\longrightarrow} P$ are allowed. Therefore, the above meaning $\llbracket P \rrbracket^{@}$ can be a non-wellfounded set.

Traditionally, this 'problem' has been overcome by imposing either an order or a metric on the transition relation, and then defining $\llbracket P \rrbracket^{@}$ as a suitable limit. Instead, in Aczel (1988) a more radical approach is taken and the standard foundation axiom is replaced by the 'anti-foundation axiom', which allows for non-well-founded sets. (See also the equivalent ' $X_{1}$-axiom' in Forti and Honsell (1983).)

One of the contributions of the present work is to show that the anti-foundation axiom is the dual, in a formal sense, of the foundation axiom. Indeed, we prove that foundation is equivalent to postulating that the universe of sets is an initial algebra for a suitable power-set endofunctor' and, dually, that anti-foundation is equivalent to postulating that the universe of sets is a final coalgebra (for the same power-set endofunctor).

From a categorical point of view one does not need to resort to non-standard foundations: as is already clear in Aczel (1988), coinductive definitions can be founded on final coalgebras, and these also exist in the standard category of ordinary sets (and in many other categories). What the anti-foundation axiom gives is the non-standard fact that the greatest (strict) fixed point

$$
g f p(F)=F(g f p(F))
$$

of an endofunctor $F$ on the category SET of classes (i.e., large sets) is a final $F$-coalgebra, provided $F$ satisfies some mild conditions. This theorem (Aczel 1988, 'Special Final Coalgebra Theorem') is the 'dual' of the standard fact (which also holds without antifoundation) that the least fixed points of most endofunctors on SET are initial algebras. 
The Special Final Coalgebra Theorem is stated in terms of the 'Solution Lemma' (Aczel 1988). The final coalgebra presentation of anti-foundation introduced here makes the Solution Lemma (and its equivalence with anti-foundation) trivial. Correspondingly, the 'uniformity on maps' condition - which an endofunctor has to satisfy in order for the Special Final Coalgebra Theorem to hold - can be formulated here somewhat more transparently than in Aczel (1988).

Aczel's semantics of CCS exploits the Special Final Coalgebra Theorem by noticing that the intended operational model of CCS can be seen as a coalgebra of the endofunctor mapping a class $X$ to the class $\mathscr{P}_{S}($ Act $\times X)$ having as elements (small) sets of pairs $\langle a, x\rangle$, with $a \in A c t$ and $x \in X$, where Act is the set of actions that can be performed by the programs. The carrier of this operational model is the set Prog of programs, and the coalgebra structure is the function $\llbracket-\rrbracket:$ Prog $\rightarrow \mathscr{P}_{S}($ Act $\times$ Prog $)$ defined for every program $P \in$ Prog as the set of pairs $\left\langle a, P^{\prime}\right\rangle$ such that $P$ can perform an action $a$ and become $P^{\prime}$ :

$$
\llbracket P \rrbracket=\left\{<a, P^{\prime}>\mid P \stackrel{a}{\longrightarrow} P^{\prime}\right\} .
$$

The function $\llbracket-\rrbracket^{@}$ mapping a program to its abstract meaning can then be defined as the coinductive extension of this coalgebra structure, that is, as the unique coalgebra homomorphism from the intended operational model to the greatest fixed point of the 'behaviour endofunctor'

$$
B X=\mathscr{P}_{S}(\text { Act } \times X),
$$

which, by the Special Final Coalgebra Theorem, is a final coalgebra:

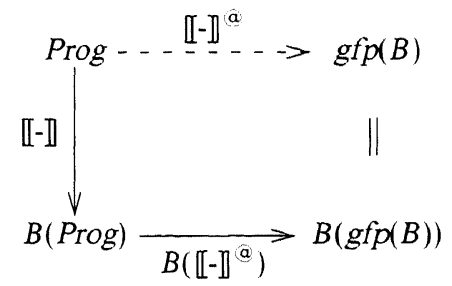

That is, for every program $\left.P, \llbracket P \rrbracket^{@}=\left\{<a, \llbracket P^{\prime} \rrbracket^{@}\right\rangle \mid P \stackrel{a}{\longrightarrow} P^{\prime}\right\}$.

\subsection{Final coalgebra semantics}

The above semantics for CCS and its properties can be generalised to arbitrary behaviours, leading to what is called here the final coalgebra approach to semantics: given a coalgebraic operational model for a programming language, its coinductive extension the final coalgebra semantics of the language - is a semantics that is fully abstract with respect to the behaviour, in the sense that two programs are identified if and only if they are behaviourally equivalent.

The basic idea is that a good, mathematical notion of behaviour should correspond to an endofunctor $B$, whose coalgebras should give the operational models for $B$. In particular, final coalgebras are canonical domains of interpretation. Indeed, as shown here, final coalgebras are 'strongly extensional', that is, two elements of the final $B$-coalgebra are equal if and only if they are ' $B$-bisimilar'. The latter is a coalgebraic generalization given in 
Aczel and Mendler (1989) of the notion of strong bisimilarity used in concurrency theory (Park 1981); it subsumes other behavioural equivalences as, for example, applicative bisimulation and trace equivalence - cf. Fiore (1996b) and Rutten and Turi (1994). Thus, semantically, the final coalgebra of a behaviour endofunctor, if it exists, is an operational model where behaviourally equivalent elements are indistinguishable. In other words, the operational model given by finality is internally fully abstract with respect to $B$.

Since the coalgebra homomorphisms are morphisms between the carriers of the operational models that 'respect' the behaviour, the coinductive extension of an operational model identifies two elements if they are behaviourally equivalent in the above sense. Under the additional hypothesis that the behaviour $B$ preserves 'weak pullbacks', coinduction can be 'pulled back' to coalgebraic bisimulation. The converse also holds, that is, two elements are identified if and only if they are behaviourally equivalent.

This final coalgebra approach to semantics complements the standard initial algebra approach (Goguen et al. 1978), where, given a functorial notion of signature $\Sigma$ for a language, the programs are described as the initial $\Sigma$-algebra, the most concrete denotational model; the other $\Sigma$-algebras are denotational models whose structure can always be inductively extended, by initiality, to a compositional interpretation of the programs. A suitable interplay between initial algebra and final coalgebra semantics is the basis of the categorical approach to structural operational semantics presented in Turi and Plotkin (1997) - see also Turi (1997) and Turi (1996).

\subsection{Partial orders}

Another way of looking at initial algebras and final coalgebras of endofunctors $F$ is as data types: the initial $F$-algebra is the inductive data type corresponding to the 'type constructor' $F$, while the final $F$-coalgebra is the coinductive one. For instance, the type constructor $F X=1+X$ yields, in the category Set of sets and functions, the natural numbers $N$ as inductive data type and the 'extended natural numbers' $N \cup\{\infty\}$ as coinductive data type.

Studies on coinductive types in Set date back at least to Arbib and Manes (1980). A more recent view, put forward by Peter Freyd in Freyd (1991), is that data types should be defined in algebraically compact categories, that is, in categories where endofunctors have both initial algebras and final coalgebras, which, moreover, do coincide in the sense that they are 'canonically isomorphic' - see also Freyd (1990) and Freyd (1992). This gives a useful mixed induction-coinduction principle - cf. Pitts (1994a) and Pitts (1994b).

One of the main examples of algebraically compact categories is the category Cppo $\perp_{\perp}$ of complete pointed partial orders and strict continuous functions: regarded as an "orderenriched' category, it has as endofunctors the 'locally continuous' ones, which, as shown in Smyth and Plotkin (1982), do indeed make it algebraically compact.

In the present study, an order-enriched version of the final coalgebra semantics approach is also introduced. A preliminary version of this work contained a definition of coalgebraic bisimulation in the order-enriched setting. Such a definition has been refined in Fiore (1996b), together with the theorem stating that final coalgebras of locally continuous endofunctor on $\underline{\mathrm{Cppo}}_{\perp}$ are strongly extensional with respect to this ordered 
coalgebraic bisimulation. This is generalized here to a full abstraction result, which, in particular, can be applied to the final coalgebra semantics of non-terminating programs.

Notice that algebraic compactness is one of the axioms of Fiore and Plotkin's axiomatic domain theory (Fiore and Plotkin 1992; Fiore and Plotkin 1994; Fiore 1996a), which aims to isolate the abstract properties that a category should satisfy for hosting interpretations of programming languages. In particular, the semantic domain of a language - in the present setting the final coalgebra of the behaviour - should 'live' in such a category, typically Cppo $_{\perp}$.

The category of sets, whether ordinary or non-well-founded, is not algebraically compact (because algebraic compactness is inconsistent with cartesian closure). Still, a naive semantics in Set is often a good starting point for more refined analyses, especially in settings where, as in concurrency, partiality does not play a central role.

\subsection{Metric spaces}

With partial orders, one can assess not only the semantic equality or inequality of programs, but also whether a program is semantically 'better' than another. Instead of such 'qualitative' judgements, one might be interested in a 'quantitative' relation between programs, with the semantics giving the distance between each pair of programs. This leads to the use of metric spaces in semantics - see de Bakker and Rutten (1992) and de Bakker and de Vink (1996) for overviews.

The main mathematical tool available in (complete) metric spaces is 'Banach's theorem', which ensures the existence of unique fixed points of 'contractive' endofunctions. Banach's theorem, especially in its higher-order form, can be used to deal with coinduction. Here it is proved that the unique fixed point of a 'locally contractive' endofunctor as given by Banach's theorem is both a final coalgebra and an initial algebra of that endofunctor. This shows that the category $\underline{\mathrm{Cms}}$ of complete metric spaces and non-expansive functions, with as endofunctors the locally contractive ones, is, like $\underline{C p p o}_{\perp}$, algebraically compact.

Structures where both the quantitative and qualitative information are present are the quasimetric spaces studied in Smyth (1988) and Bonsangue et al. (1998). These are metric spaces where distances might be non-symmetric. Such an asymmetry requires a more subtle notion of limit than for metric spaces, for which contractive functions need not be continuous. But once these differences are taken into account, Banach's theorem also holds in this setting, and the algebraic compactness theorem for $\mathrm{Cms}$ carries over to the category Cqms of complete quasimetric spaces and functions that are both non-expansive and continuous.

A further generalization is achieved in Wagner (1994) (see also Flagg and Kopperman (1997)), where structures parametric in a 'quantale' $\Omega$ are studied. In particular, generalized notions of Cauchy sequence and limit are given, and, at a higher level, they are used to show that the standard constructions of final coalgebras (alias initial algebras) in $\underline{\mathrm{Cms}}$ and in $\underline{\mathrm{Cppo}}_{\perp}$ are both instances of one construction parametric in $\Omega$. The use of $\Omega$ stems from a foundational article by Lawvere (1973) presenting metric spaces, partial orders and categories as instances of one and the same notion. 
Structure of the paper

Section 2 contains the definitions of algebraic induction and coalgebraic coinduction. Also, examples of operational models as coalgebras of behaviour endofunctors are given. In Section 3, it is shown that initial algebras and final coalgebras can both be seen as adjoints to suitable forgetful functors. Following Barr (1993), this fact is used to show that the finite power-set functor has a final coalgebra.

In Section 4, the coalgebraic notion of bisimulation introduced in Aczel and Mendler (1989) is studied. Several properties are proved that generalize standard results on (ordinary) bisimulations. In particular, we prove a general full abstraction theorem stating that in final coalgebra semantics two programs are identified if and only if they are behaviourally equivalent. Next, we show how to obtain final coalgebras from weakly final ones using coalgebraic bisimulations. Finally, we introduce a new notion of bisimulation, which is parametric in the possible transformations of states.

Section 5 is devoted to a coalgebraic presentation of Peter Aczel's theory of nonwell-founded sets. The main novelty is that a categorical duality is proved between the anti-foundation axiom (giving non-well-founded sets) and the standard foundation axiom. Also, simplified presentations of the 'Solution Lemma', of the 'substitution lemma' and of the notion of a functor uniform on maps are given. This allows for a more transparent proof of the 'Special Final Coalgebra Theorem', one of the most relevant results, from a semantical point of view, in Aczel (1988).

Section 6 gives a survey of final coalgebras and their bisimulations in order-enriched categories; it is based on material in Smyth and Plotkin (1982) and Fiore (1996b). It also contains a full abstraction theorem with respect to the order-enriched version of coalgebraic bisimulation.

The last section is dedicated to final coalgebras in categories of metric spaces. It is shown that the categorical version of Banach's fixed point theorem introduced in America and Rutten (1989) yields a final coalgebra (canonically isomorphic to an initial algebra). Also, the above mentioned recent results on generalized notions of metric spaces, which reconcile the order-theoretic with the metric-theoretic approach, are discussed.

\section{From algebraic induction to coalgebraic coinduction}

The most elementary form of induction, the one on the natural numbers

$$
N=\left\{0,1=s(0), 2=s^{2}(0), \ldots\right\},
$$

is based on the following theorem.

Theorem 2.1. (Recursion Theorem) Given a set $X$, an element $e \in X$ and a function $g: X \rightarrow X$, there exists a unique function $f: N \rightarrow X$ from the set of natural numbers to the given set such that

$$
f(0)=e \quad \text { and } \quad f(s(n))=g(f(n))
$$

for all numbers $n \in N$. 
The value $e$ of the function $f$ at (the least element) 0 (with respect to the order relation) is the 'base' of the induction, and $g$ defines the 'inductive step'.

The Recursion Theorem can be taken as the definition of natural numbers. That is, every set $N$ with a distinguished element $0 \in N$ and a unary operation $s: N \rightarrow N$ such that the Recursion Theorem holds, is isomorphic to the natural numbers - see, for example, Mac Lane (1986, Chapter 2). As pointed out by Lawvere, the existence/uniqueness statement of the Recursion Theorem asserts the universal property characterizing the natural numbers: initiality. This property underlies induction, not only on the natural numbers, but in general.

Initiality is the most elementary universal property that an object of a category can enjoy: an object $X$ is initial in a category if, for every object $Y$ of the category, there exists a morphism $f: X \rightarrow Y$ from $X$ to $Y$, and, moreover, this morphism is unique.

The basic way of understanding the natural numbers as an initial object is to regard them as an object $\langle N, 0, s\rangle$ in the category having as objects triples $\langle X, e, t\rangle$, where $X$ is a set with a distinguished element $e \in X$ and a function $t: X \rightarrow X$ on it. The morphisms $f:\langle X, e, t\rangle \rightarrow\left\langle X^{\prime}, e^{\prime}, t^{\prime}\right\rangle$ of the category are functions $f: X \rightarrow X^{\prime}$ such that

$$
f(e)=e^{\prime} \quad \text { and } \quad f(t(x))=t^{\prime}(f(x)) .
$$

(It is easy to verify that the above objects and morphisms form a category with composition and identities as in Set.) Then the Recursion Theorem says exactly that the triple $\langle N, 0, s\rangle$ is initial in this category. (Notice that in the category Set, the initial object is the trivial empty set.) Conversely, since initial objects are unique up to isomorphism (as are all universals), the initial object of this category defines the natural numbers up to isomorphism.

This specific form of induction can be generalized through the following series of abstractions.

First, notice that the element $e \in X$ of a set $X$ can be written as a function from the one-element set $1=\{*\}$ to the set $X$; that is, one can identify a function $e: 1 \rightarrow X$ from the one-element set 1 to a set $X$ with its value $e(*) \in X$ at the unique element $*$ of 1 . Then the Recursion Theorem amounts to having an object $1 \stackrel{0}{\longrightarrow} N \stackrel{s}{\longrightarrow} N$ such that for every object $1 \stackrel{e}{\longrightarrow} X \stackrel{g}{\longrightarrow} X$, there exists a unique function $f: N \rightarrow X$ with

$$
f \circ 0=e \quad \text { and } \quad f \circ s=g \circ f
$$

Diagrammatically, using dashed morphisms to denote morphisms given by universal properties, one has that the following diagram commutes:

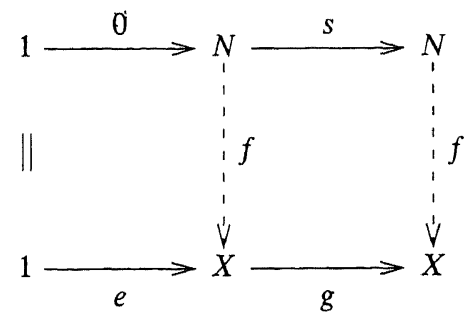

Second, every pair of functions with the same codomain (thus, for example, $e: 1 \rightarrow X$ and $g: X \rightarrow X$ ) can be made into a single morphism with as domain the disjoint union of 
the domains. This holds in general in every category with coproducts: given two objects $X$ and $Y$ in a category, their coproduct, if it exists, is an object $X+Y$ with two morphisms inl $_{X}: X \rightarrow X+Y$ and inl $Y: Y \rightarrow X+Y$, which is universal in the sense that for every pair of morphisms $f: X \rightarrow Z$ and $f: Y \rightarrow Z$ there exists a unique morphism $[f, g]: X+Y \rightarrow Z$, making the following diagram commute:

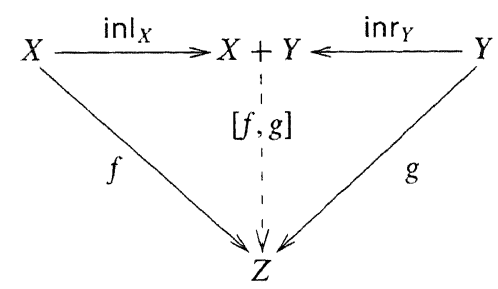

(The dual of the coproduct $X+Y$ is the product $X \times Y:$ its projections fst $X: X \times Y \rightarrow X$ and snd $: X \times Y \rightarrow Y$ are universal among all pairs of morphisms $f: Z \rightarrow X$ and $g: Z \rightarrow Y$.)

In Set, the disjoint union, together with the corresponding injection functions, is a coproduct. Hence, one can write $[e, g]: 1+X \rightarrow X$ instead of $1 \stackrel{e}{\longrightarrow} X \stackrel{g}{\longrightarrow} X$. Correspondingly, the initiality of the natural numbers can be expressed by saying that for every function $h: 1+X \rightarrow X$ there exists a unique morphism $f: N \rightarrow X$ such that the following diagram commutes:

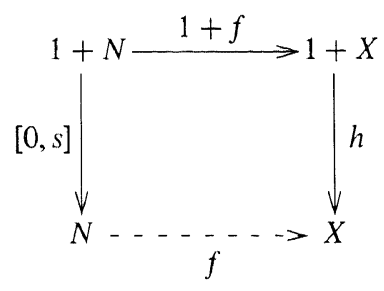

The morphism $1+f: 1+N \rightarrow 1+X$ is defined by universality: using id $_{X}$ to denote the identity morphism on an object $X$,

$$
1+f=\left[\operatorname{inl}_{1} \circ \operatorname{id}_{1}, \operatorname{inr}_{X} \circ f\right]=\left[\operatorname{inl}_{1}, \operatorname{inr}_{X} \circ f\right]: 1+N \rightarrow 1+X .
$$

Thus the operation $X \mapsto 1+X$ on objects extends to an operation $f \mapsto 1+f$ on morphisms: this defines a functor from $\underline{\text { Set }}$ to $\underline{\text { Set}}$, that is, an endofunctor on $\underline{\text { Set. }}$.

The third step of abstraction is now to move from the above (endo) functor $F X=1+X$ on $\underline{\text { Set }}$ to arbitrary endofunctors $F$ on arbitrary categories $\underline{\mathrm{C}}$ and, correspondingly, to consider initial objects in categories of structures $h: F X \rightarrow X$ rather than $h: 1+X \rightarrow X$.

Given an endofunctor $F: \underline{C} \rightarrow \underline{C}$ on a category $\underline{C}$, one can form the category $\underline{C}^{F}$ of $F$-algebras having as objects pairs $\langle X, h\rangle$ with $X$ an object and $h: F X \rightarrow X$ a morphism

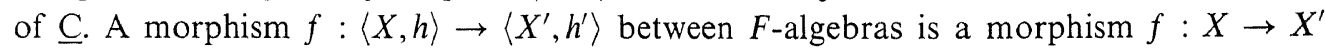


between their 'carriers' such that

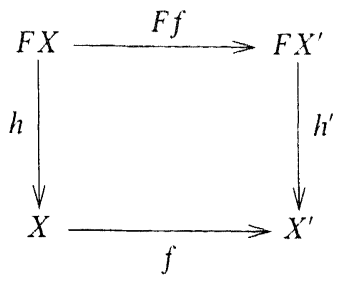

commutes, that is, $f \circ h=h^{\prime} \circ F f$. Therefore, the natural numbers can also be understood as the initial algebra of the endofunctor $F X=1+X$ on Set.

The initial object in the category of algebras of an arbitrary endofunctor $\Sigma$ (that is, the initial $\Sigma$-algebra) does not always exists, but if it does, its structure is an isomorphism, as given by the following lemma.

Lemma 2.1. (Lambek's Lemma: initial algebras are isomorphisms) Let $\langle\bar{F}, \psi\rangle$ be the initial algebra of an arbitrary endofunctor $\Sigma$. Then the algebra structure $\psi: F \bar{F} \rightarrow \bar{F}$ is always an isomorphism

$$
\psi: F \bar{F} \cong \bar{F} \quad \text { (initial } F \text {-algebra). }
$$

(To prove this notice that the initial algebra structure $\psi$ is also an $F$-algebra morphism from $\langle F \bar{F}, F \psi\rangle$ to $\langle\bar{F}, \psi\rangle$.)

As mentioned in the Introduction, initial algebras give a very useful induction principle. Indeed, every algebra structure $h: F X \rightarrow X$ of an arbitrary endofunctor $F$ with initial algebra $F \bar{F} \cong \bar{F}$ can be inductively extended to a morphism $h^{*}: \bar{F} \rightarrow X$ by taking the unique algebra morphism from the initial algebra to the algebra $\langle X, h\rangle$ :

\section{Inductive Extension}

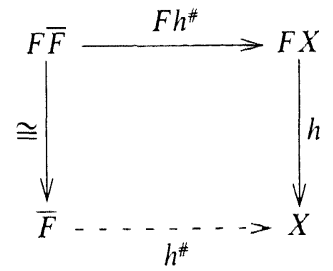

Notice that initiality gives both the existence of inductive extensions and their uniqueness. The former accounts for the definitional power of induction, while the latter gives a proof principle, since one can prove two morphisms equal by showing that both fit as inductive extensions of an algebra structure.

\subsection{Coalgebras}

The dual of the notion of initiality is the notion of finality: an object $X$ is final (or terminal) in a category when from every object of the category there is a unique morphism to $X$. And the dual of the notion of an algebra of an endofunctor $F$ on a category $C$ is the notion of an $F$-coalgebra, that is, a pair $\langle X, k\rangle$ with $X$ an object and $k: X \rightarrow F X$ a morphism of $\underline{C}$. The coalgebras of an endofunctor $F: \underline{C} \rightarrow \underline{C}$ are the objects of the 
category $\underline{\mathrm{C}}_{F}$ of $F$-coalgebras having as morphisms $f:\langle X, k\rangle \rightarrow\left\langle X^{\prime}, k^{\prime}\right\rangle$ those morphisms $f: X \rightarrow X^{\prime}$ between the carriers such that

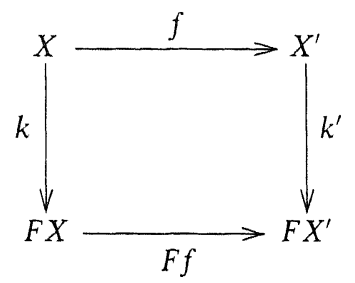

commutes, that is, $F f \circ k=k^{\prime} \circ f$.

The final object of a category, as initial objects, coproducts, pullbacks, and all other universals, is unique up to isomorphisms. Therefore, one often speaks of the final coalgebra instead of $a$ final coalgebra.

\subsection{Operational models as coalgebras of behaviours}

As mentioned in the Introduction, the semantic relevance of coalgebras is that they can be used to represent operational models. Let us write $B$ (behaviour) for endofunctors whose coalgebras have a computational interest.

The main example of such a functorial notion of behaviour is the endofunctor

$$
B X=\mathscr{P}(A c t \times X)
$$

mapping a set $X$ to the set of pairs $\langle a, x\rangle$, with $a$ in Act and $x$ in $X$. Its coalgebras $\langle X, k\rangle$ can be seen as labelled transition systems (Plotkin 1981), that is, triples $\langle X, A c t, \rightarrow\rangle$, where $X$ is a set of 'states', Act is a set of 'labels' and $\rightarrow \subseteq X \times A c t \times X$ is a 'transition' relation. The bijection between coalgebras $\langle X, k\rangle$ of the above behaviour and labelled transition systems $\langle X, A c t, \rightarrow\rangle$ is given by the following correspondence:

$$
x \stackrel{a}{\longrightarrow} x^{\prime} \Longleftrightarrow<a, x^{\prime}>\in k(x) .
$$

Labelled transition systems are operational models for non-deterministic (and concurrent) languages with atomic actions. The transition $x \stackrel{a}{\longrightarrow} x^{\prime}$ in the model says that the state $x$ can perform an action $a$ and reach the state $x^{\prime}$. The natural morphisms of transition systems are functions $f: X \rightarrow X^{\prime}$ between their sets of states that preserve the transition relation. That is, if $x \stackrel{a}{\longrightarrow} x^{\prime}$, then $f(x) \stackrel{a}{\longrightarrow} f\left(x^{\prime}\right)$. Coalgebraically, this accounts for only one half of the equality $B f \circ k=k^{\prime} \circ f$ that has to hold for a coalgebra homomorphism, namely the inclusion

$$
B f \circ k \subseteq k^{\prime} \circ f .
$$

The reverse inclusion amounts to the condition that if $f(x) \stackrel{a}{\longrightarrow} y$ for some $y$ in $X^{\prime}$, then there exists a state $x^{\prime}$ in $X$ such that $x \stackrel{a}{\longrightarrow} x^{\prime}$ and $f\left(x^{\prime}\right)=y$. Morphisms of transition systems that verify this extra condition are exactly those whose graph is a strong bisimulation. They are also known in the literature as the 'zig-zag morphisms' (van Benthem 1984) (or as the 'saturating morphisms' (Arnold and Dicky 1989)), which, as 
shown in Joyal et al. (1993), are the ' $\underline{\mathrm{P}}$-open morphisms', for a suitable subcategory $\underline{\mathrm{P}}$ of the category of transition systems - cf. the next section.

Several interesting variations of the above behaviour are obtained by varying the choice of power-set endofunctor. For instance, by taking the restriction of $\mathscr{P}$ to the finite power-set $\mathscr{P}_{f}$, one has the behaviour

$$
B X=\mathscr{P}_{f}(A c t \times X),
$$

whose coalgebras are the finitely branching transition systems, with states each able to choose among a finite set of transitions. Similarly, the endofunctor $\mathscr{P}_{S}$ on the category SET of classes (that is, large sets) that maps a class to the class of its subsets (thus small sets) gives the behaviour

$$
B X=\mathscr{P}_{S}(\text { Act } \times X),
$$

whose coalgebras are the 'locally small transition systems', that is, transition systems possibly having a large set (that is, a class) of states, each able to choose among a (small) set of transitions.

The above notions of behaviour all account for non-deterministic transition systems. For the simpler case of deterministic systems, where each state can perform at most one transition, one can consider the behaviour endofunctor

$$
B X=1+\text { Act } \times X \text {. }
$$

A coalgebra structure $k: X \rightarrow 1+$ Act $\times X$ maps a state $x$ either to the only element ' $*$ ' of the singleton set $1=\{*\}$ (that is, $x$ cannot perform any transition) or to a pair $\left\langle a, x^{\prime}\right\rangle$ (that is, $x \stackrel{a}{\longrightarrow} x^{\prime}$ ). One can check that the set $A c t^{\infty}=A c t^{*} \cup A c t^{\prime \nu}$ of finite and infinite words over the alphabet $A c t$ is the carrier of a final coalgebra for this behaviour.

The above behaviours, whose coalgebras correspond to various forms of labelled transition systems, are suitable for modelling imperative and concurrent languages. Instead, for modelling applicative languages, one usually needs behaviours involving some form of function space functor. An example is the endofunctor

$$
B X=1+X^{Y} .
$$

The 'exponent' $X^{Y}$ is the set of functions from $Y$ to $X$. In order to avoid the usual 'mixed variance' problems, $Y$ is here treated as a parameter. By putting $Y=X$ one obtains that the corresponding coalgebras are the quasi-applicative transition systems defined in Abramsky (1990). The 'exception' 1 in the above behaviour can be used to encode nontermination. For example, for $X$ and $Y$ both equal to the set $\Lambda$ of closed $\lambda$-terms, one can define a coalgebra structure

$$
e v: \Lambda \rightarrow 1+\Lambda^{\Lambda}
$$

by putting, for every $\lambda$-term $M \in \Lambda$,

$$
\operatorname{ev}(M)=P \mapsto N[P / x]
$$

if $M$ converges to 'principal weak head normal form' $\lambda x . N$, and

$$
\operatorname{ev}(M)=*
$$

otherwise. 
Examples of operational models as coalgebras in categories of partial orders and of metric spaces are given in Sections 6 and 7. Many more functorial notions of behaviour are currently under investigation.

\subsection{Coinduction}

Let $F$ be an endofunctor that has a final coalgebra (that is, the final object in the corresponding category of coalgebras) and let $\widehat{F}$ denote the carrier of this final coalgebra. The structure $\varphi$ of a final coalgebra is, like that of an initial algebra, an isomorphism (Lemma 2.1), because the notion of isomorphism is 'self-dual'. Thus we have the following observation.

Observation 2.1. (Final coalgebras are isomorphisms) Let $\langle\widehat{F}, \varphi\rangle$ be the final coalgebra of an arbitrary endofunctor $F$. Then the coalgebra structure $\varphi: F \widehat{F} \rightarrow \widehat{F}$ is always an isomorphism

$$
\varphi: \widehat{F} \cong F \widehat{F} \quad \text { (final } F \text {-coalgebra). }
$$

Notice, that from cardinality, this implies that the (unrestricted) power-set endofunctor $\mathscr{P}$ cannot have a final coalgebra, and the same holds for the behaviour $B X=\mathscr{P}(A c t \times X)$. However, final coalgebras for the variations of these endofunctors considered above do exist. As illustrated by Corollary 3.1, the finite power-set endofunctor $\mathscr{P}_{f i}: \underline{\text { Set }} \rightarrow \underline{\text { Set }}$ has a final coalgebra and similar arguments show that the power-set endofunctor $\mathscr{P}_{S}: \underline{\mathrm{SET}} \rightarrow$ $\underline{\text { SET }}$ on the category of classes and the behaviour endofunctors $B X=\mathscr{P}_{f}($ Act $\times X)$ and $B X=\mathscr{P}_{S}($ Act $\times X)$ also have final coalgebras.

Any coalgebra structure $k: X \rightarrow F X$ can be 'coinductively' extended to a morphism $k^{(\mathrm{a}}: X \rightarrow \widehat{F}$ by taking the unique coalgebra morphism from the coalgebra $\langle X, k\rangle$ to the final coalgebra:

\section{Coinductive Extension}

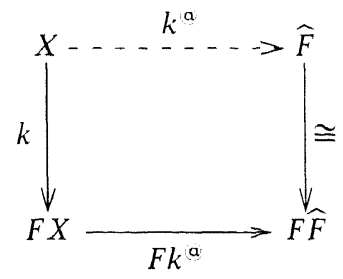

For example, by taking for $F$ the behaviour $B X=\mathscr{P}_{S}(A c t \times X)$ and for $k: X \rightarrow F X$ the coalgebra structure $\llbracket-\rrbracket:$ Prog $\rightarrow B($ Prog) corresponding to the intended operational model for CCS, as mentioned in the Introduction, the coinductive extension $\llbracket-\mathbb{-}^{\mathrm{a}}: \operatorname{Prog} \rightarrow \widehat{B}$ is a final coalgebra semantics: for every program $P$ of $\mathrm{CCS}$,

$$
\llbracket P \rrbracket^{(a)}=\varphi^{-1}\left\{<a, \llbracket P^{\prime} \rrbracket^{\mathfrak{a}}>\mid P \stackrel{a}{\longrightarrow} P^{\prime}\right\},
$$

where $\varphi^{-1}$ is the inverse of the final coalgebra isomorphism $\varphi: \widehat{B} \cong B \widehat{B}$ given by (the dual of) Lambek's lemma (Observation 2.1). If one postulates the anti-foundation axiom, the (strict) greatest fixed point $g f p[B]=B(g f p[B])$ of the above $B$ is a final coalgebra, 
and Equation (2) is strict, that is, the isomorphism $\varphi$ cuts down to an identity:

$$
\llbracket P \rrbracket^{a}=\left\{<a, \llbracket P^{\prime} \rrbracket^{a}>\mid P \stackrel{a}{\longrightarrow} P^{\prime}\right\} .
$$

\section{Adjunctions subsume induction and coinduction}

Induction and coinduction can be made parametric in a suitable sense by considering the more general notions of 'free algebras' and 'cofree coalgebras' instead of initial algebras and final coalgebras only. This gives a natural way of understanding initial algebra and final coalgebra constructions as 'canonical' categorical constructions, namely as adjoint to suitable elementary functors, which allows one to solve the problem of establishing the existence of initial algebras and final coalgebras by means of general categorical theorems, such as the 'Special Adjoint Functor Theorem'. Following Barr (1993), this is done here, in particular, for the coalgebras of the (finite) power-set functor. Moreover, the more general induction and coinduction principles available from free algebras and final coalgebras, respectively, are shown to be founded on the ubiquitous categorical notion of an adjunction.

\subsection{Free algebras of terms}

In categories with binary coproducts, the free algebra of an endofunctor $F$ over an object $X$ is the initial algebra of the endofunctor $X+F$, mapping an object $Y$ to the coproduct $X+F Y$. Let us illustrate this by considering the free algebras over sets generated by a signature $\Sigma$, that is a set of function symbols $\sigma$ and a corresponding 'arity' function.

The carrier of a free algebra over a set $X$ generated by a signature $\Sigma$ is the set $T X$ of terms with variables $x$ in $X$ generated by $\Sigma$. Categorically, a signature can be seen as an operator

$$
X \mapsto \coprod_{\sigma \in \Sigma} X^{\operatorname{ar}(\sigma)}
$$

mapping a set $X$ to the coproduct, indexed by the symbols in $\Sigma$, of powers $X^{\operatorname{ar}(\sigma)}$ of $X$. For instance, for the signature $\{0, s\}$ corresponding to the natural numbers, which consists of a constant (that is, 0 -ary symbol) and a unary symbol, one has $X \mapsto X^{0}+X^{1}=1+X$.

For every signature $\Sigma$ this operator extends to an endofunctor $\Sigma: \underline{\text { Set }} \rightarrow \underline{\text { Set }}$ in the obvious way. The initial $\Sigma$-algebra exists and its carrier $\bar{\Sigma}$ is the set $T 0$ of closed terms generated by $\Sigma$.

More generally, for every set $X$, the endofunctor

$$
(X+\Sigma): \underline{\text { Set }} \rightarrow \underline{\text { Set }}
$$

with action $Y \mapsto X+\Sigma Y$, has an initial algebra with, as carrier, the set $T X$ of terms with variables $x \in X$. Since, by Lambek's lemma, initial algebras are isomorphisms

$$
X+\Sigma T X \cong T X
$$

the set $T X$ is a coproduct and its algebra structure is the copair of the injections

$$
\text { inl }_{X}: X \rightarrow T X \quad \operatorname{inr}_{X}: \Sigma T X \rightarrow T X .
$$


The left injection is the usual insertion of variables $x \in X$ into the terms $t \in T X$, which is usually left implicit. Formally, $x$ is simply an element of the set $X$ and it is only after applying inl $X$ to it that one obtains a variable. This variable-making function is usually written as

$$
\eta_{X}=\operatorname{inl}_{X}: X \rightarrow T X
$$

The other injection $\operatorname{inr}_{X}: \Sigma T X \rightarrow T X$ is the operation that permits one to construct a new term given any $n$-ary operator $\sigma$ and terms $t_{1}, \ldots, t_{n}$. The right injection is usually left implicit and one simply writes $\sigma\left(t_{1}, \ldots, t_{n}\right)$ for the resulting term.

As $T 0$ and $T X$ are initial algebras, they come with an induction principle, which, since $T X$ is a coproduct, can be rephrased as follows: for every $\Sigma$-algebra structure $h: \Sigma Z \rightarrow Z$ and every 'valuation' function $f: X \rightarrow Z$ of the variables in $X$ as elements of the algebra $\langle Z, h\rangle$, there exists a unique function $f^{\#}: T X \rightarrow Z$ making

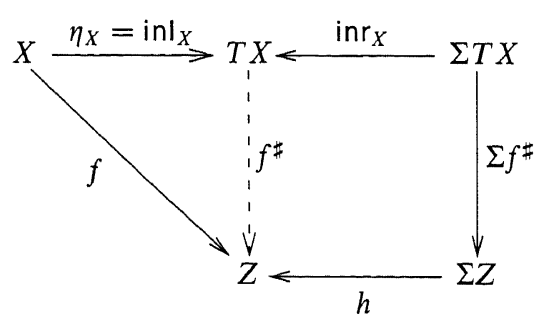

commute. Omitting the injections,

$$
f^{\sharp}(x)=f(x) \quad \text { and } \quad f^{\sharp}\left(\sigma\left(t_{1}, \ldots, t_{n}\right)\right)=h\left(\sigma\left(f^{\sharp}\left(t_{1}\right), \ldots, f^{\sharp}\left(t_{n}\right)\right)\right) .
$$

This inductive extension of $h$ along the valuation function $f$ is, formally, the inductive extension $[f, h]^{\#}$ of the $(X+\Sigma)$-algebra structure on $Z$ given by the copair

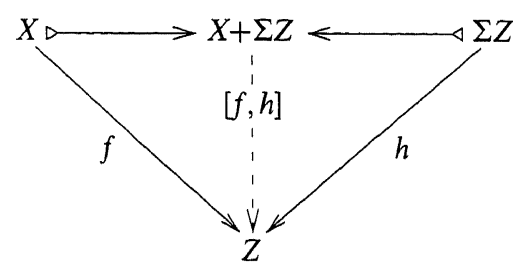

As an example, this induction principle can be used to show that the operator $T$

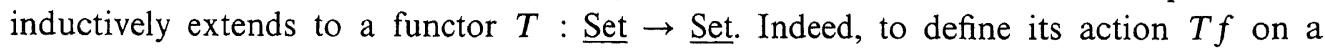
function $f: X \rightarrow Y$, take the inductive extension of $\operatorname{inr}_{Y}: \Sigma T Y \rightarrow T Y$ along the 
composite inl $\mathrm{I}_{Y} \circ \mathrm{f}$ :

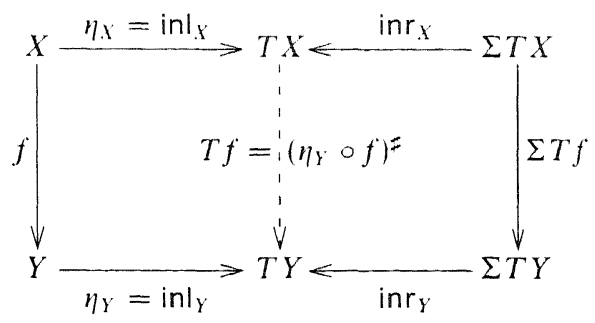

To prove that this definition is functorial, that is, $T\left(\mathrm{id}_{X}\right)=\mathrm{id}_{T X}$ and $T(g \circ f)=T g \circ T f$, for $g: Y \rightarrow Z$, one exploits the uniqueness of inductive extensions: the function $i_{T X}$ fits as $\left(\eta_{X} \circ \mathrm{id}_{X}\right)^{\#}=\left(\eta_{X}\right)^{\#}$ and $T g \circ T f$ fits as $\left(\eta_{Z} \circ g \circ f\right)^{\#}$.

Notice that a function $f: X \rightarrow Y$ can be seen as a 'renaming' of variables, and then the function $T f: T X \rightarrow T Y$ is the inductive extension of such a renaming from simple variables to complex terms with variables.

\subsection{Adjunctions}

The existence of an inductive extension $f^{\#}: T X \rightarrow Z$ of every algebra structure $h$ : $\Sigma Z \rightarrow Z$ along any function $f: X \rightarrow Z$ shows that there is a bijection between $\Sigma$-algebra morphisms of type $\left\langle T X, \operatorname{inr}_{X}\right\rangle \rightarrow\langle Z, h\rangle$ and functions of type $X \rightarrow Z$ :

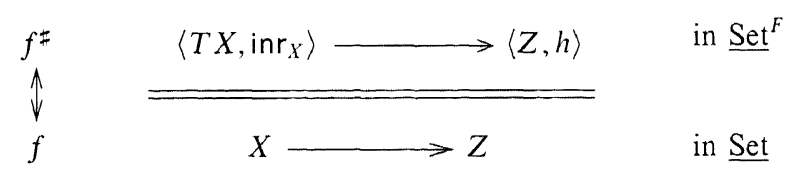

Moreover, this bijection is natural in $X$ and $\langle Z, h\rangle$. This says that the two (operations extending to) functors $X \mapsto\left\langle T X\right.$,inr $\left.r_{X}\right\rangle$ and $\langle Z, h\rangle \mapsto Z$ are adjoint - see, for example, Mac Lane (1971, page 78) for the definition of adjoint functors.) The former is the functor from Set to $\underline{\operatorname{Set}^{\Sigma}}$ mapping a set to the free $\Sigma$-algebra over it; the latter is the forgetful functor

$$
U^{\Sigma}: \underline{\operatorname{Set}}^{\Sigma} \rightarrow \underline{\operatorname{Set}}
$$

mapping algebras to their carriers. The existence of free algebras is then equivalent to the existence of a left adjoint for $U^{\Sigma}$. Correspondingly, the above inductive extension $f^{\sharp}$ is called the left adjunct of $f$.

For every category of $F$-algebras such a forgetful functor

$$
U^{F}: \underline{C}^{F} \rightarrow \underline{C}
$$

exists. If it has a left adjoint

$$
F^{\star}: \underline{C} \rightarrow \underline{C}^{F}
$$

then, fo: every object $X$ in $\underline{C}, F^{*} X$ is the free $F$-algebra over $X$. (If $F=\Sigma$, then $F^{\star} X=\left\langle T X\right.$, inr $\left.{ }_{X}\right\rangle$.) For every morphism $f: X \rightarrow Z=U^{F}\langle Z, h\rangle$ in $\underline{\mathrm{C}}$, the left adjunct $f^{\#}: F^{\star} X \rightarrow\langle Z, h\rangle$ is the inductive extension of $h$ along $f$. 
Dually, for every endofunctor $F: \underline{C} \rightarrow \underline{\mathrm{C}}$, if the forgetful functor

$$
U_{F}: \underline{C}_{F} \rightarrow \underline{C}
$$

mapping a coalgebra to its carrier has a right adjoint

$$
F_{\star}: \underline{\mathrm{C}} \rightarrow \underline{\mathrm{C}}_{F}
$$

in the sense that there is a natural bijection

$$
\begin{array}{lll}
\uparrow_{f^{b}}^{f} & \stackrel{U_{F}\langle Z, k\rangle=Z \longrightarrow X}{\longrightarrow} \longrightarrow F_{\star} X & \text { in } \underline{C} \\
& \text { in } \underline{C}_{F}
\end{array}
$$

then $F_{\star} X$ is the cofree coalgebra of $F$ over $X$. The right adjunct $f^{b}$ of a morphism $f$ in $\underline{C}$ as given by the above bijection is the coinductive extension of the coalgebra structure $k: Z \rightarrow F Z$ along the morphism $f: Z \rightarrow X$.

One can check that, in categories with binary products, a cofree coalgebra of an endofunctor $F$ over an object $X$ is a final $(X \times F)$-coalgebra. Therefore, letting $\left|F_{\star} X\right|$ denote the carrier $U_{F}\left(F_{\star}(X)\right)$ of the cofree coalgebra $F_{\star} X$,

$$
\left|F_{\star} X\right| \cong X \times F\left|F_{\star} X\right| \text {. }
$$

This allows one to write the coinductive extension $f^{b}:\langle Z, k\rangle \rightarrow\left|F_{\star} X\right|$ of a coalgebra structure $k: Z \rightarrow F Z$ along a morphism $f: Z \rightarrow X$ as follows:

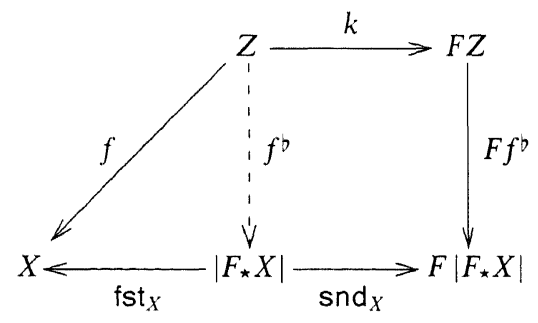

where fst $_{X}:\left|F_{\star} X\right| \cong X \times F\left|F_{\star} X\right| \rightarrow X$ and snd $_{X}:\left|F_{\star} X\right| \cong X \times F\left|F_{\star} X\right| \rightarrow F\left|F_{\star} X\right|$ are, respectively the first and second projection of the product (4). An example of such a coinductive extension is given in Section 4.4 .

One important property of right (left) adjoints is that they preserve limits (colimits) - see, for example, Mac Lane (1971, Theorem V.5.1). Therefore, if $\underline{C}$ has a final (initial) object $1(0)$, then $F_{\star} 1\left(F^{\star} 0\right)$ is a final coalgebra (initial algebra). In particular, Set has a final object, namely the singleton set, and therefore the following proposition implies that the finite power-set endofunctor $\mathscr{P}_{f i}: \underline{\text { Set }} \rightarrow \underline{\text { Set }}$ on $\underline{\text { Set }}$ has a final coalgebra.

Theorem 3.1. The forgetful functor from the category of $\mathscr{P}_{f}$-coalgebras to $\underline{\text { Set }}$ has a right adjoint.

Proof. The proof is an instance of the proof of Barr (1993, Theorem 1.2), which is stated in terms of 'accessible' endofunctors. The core of the proof is the fact that the $\mathscr{P}_{f^{-}}$-coalgebras with carrier of cardinality not greater than $\omega$ form a small 'generating set' 
$\mathscr{G}$, that is, every $\mathscr{P}_{f}$-coalgebra can be obtained by quotienting the disjoint union of a suitable set of $\mathscr{P}_{f i}$-coalgebras in $\mathscr{G}$. The existence of such a small generating set allows one to apply the 'Special Adjoint Functor Theorem'. (For more details see Turi (1996, Section 13).)

Corollary 3.1. The finite power-set endofunctor has a final coalgebra.

\section{Coalgebraic bisimulations}

There are several notions of behavioural equivalence for a transition system; the most general one corresponds to a relation on its states called (strong) bisimulation. The final coalgebra of the behaviour corresponding to transition systems 'classifies' bisimilar states in the sense that two states are bisimilar if and only if they have the same final coalgebra semantics, that is, the same abstract global behaviour. In other words, coinduction can be 'pulled back' to bisimulation. As a corollary, the final coalgebra is 'internally fully abstract'.

In this section, all this is generalized to arbitrary endofunctors preserving 'weak pullbacks'.

Given a labelled transition system $\langle X, A c t, \rightarrow\rangle$, two states $x, y \in X$ are (strongly) bisimilar if there exists a relation $R$ on the sets of states $X$ such that $x R y$ (that is, $\langle x, y\rangle \in R$ ) and 1 if $x \stackrel{a}{\longrightarrow} x^{\prime}$, then $y \stackrel{a}{\longrightarrow} y^{\prime}$ for some $y^{\prime}$ such that $x^{\prime} R y^{\prime}$

2 and, conversely, if $y \stackrel{a}{\longrightarrow} y^{\prime}$, then $x \stackrel{a}{\longrightarrow} x^{\prime}$ for some $x^{\prime}$ such that $x^{\prime} R y^{\prime}$

More generally, one can consider two transition systems with the same set Act of labels and relations $R$ between their carriers $X$ and $Y$. Then, a relation $R$ is a (strong) bisimulation between the two transition systems if, for every $x \in X$ and $y \in Y$ such that $x R y$, Clauses 1 and 2 above hold.

In terms of the coalgebraic representation (1) of transition systems, a relation $R$ between the carriers $X$ and $Y$ of two coalgebras $\langle X, k\rangle$ and $\langle Y, \ell\rangle$ is a (strong) bisimulation between the two coalgebras when, for all $x$ in $X$ and $y$ in $Y$ such that $x R y$,

- if $\left\langle a, x^{\prime}\right\rangle \in k(x)$, then $\left\langle a, y^{\prime}\right\rangle \in \ell(y)$ for some $y^{\prime}$ such that $x^{\prime} R y^{\prime}$

- and, conversely, if $\left\langle a, y^{\prime}\right\rangle \in f(y)$, then $\left\langle a, x^{\prime}\right\rangle \in k(x)$ for some $x^{\prime}$ such that $x^{\prime} R y^{\prime}$.

Notice that bisimulations are themselves coalgebras. Indeed, from the above conditions, one can define a coalgebra structure

$$
\widetilde{R}: R \rightarrow \mathscr{P}(A c t \times R)
$$

on the relation $R$ by putting (using the infix notation)

$$
\left.<a,<x^{\prime}, y^{\prime}>\right\rangle \in x \widetilde{R} y \Longleftrightarrow<a, x^{\prime}>\in k(x),<a, y^{\prime}>\in \ell(y) \text {, and } x^{\prime} R y^{\prime} .
$$

In the rest of this paper, the above notion of bisimulation is called ordinary bisimulation, in order to distinguish it from the following more general notion of "coalgebraic bisimulation'. 
Consider the two 'legs' $r_{1}: R \rightarrow X$ and $r_{2}: R \rightarrow Y$ obtained by composing the insertion $R \hookrightarrow X \times Y$ of the relation $R$ into the cartesian product $X \times Y$ with the first and second projection, respectively. If the relation $R$ is an (ordinary) bisimulation, then its legs $r_{1}$ and $r_{2}$ lift to coalgebra morphisms; that is, the two squares in

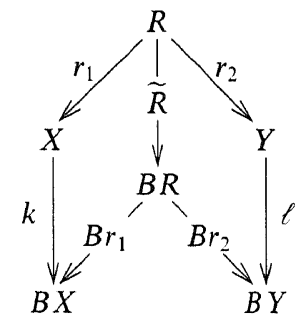

commute. The converse is also true; namely, if a relation lifts to a coalgebra of the above behaviour endofunctor $B X=\mathscr{P}(A c t \times X)$ in a way that its legs also lift to corresponding coalgebra morphisms as in the above diagram, then this relation is a bisimulation. Indeed, the first condition is obvious, while the second and the third follow from the commutativity of the left and the right diagram, respectively. Notice that there might be more structures $\widetilde{R}$ making the above diagram commute, corresponding to the several ways in which, in general, a relation can be a bisimulation.

In general, for an arbitrary endofunctor $B$ on a category $\underline{C}$, a (coalgebraic) bisimulation (or a $B$-bisimulation) between two $B$-coalgebras $\langle X, k\rangle$ and $\langle Y, \ell\rangle$ is a span

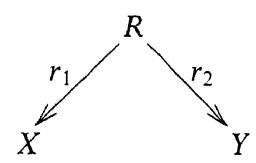

of morphisms in $\underline{C}$ such that there exists a $B$-coalgebra structure $\widetilde{R}: R \rightarrow B R$ that makes the diagram (5) commute. Notice that the stress is put on the fact that the legs of the span $R$ lift to coalgebra morphisms, rather than on the actual (possibly not unique) coalgebraic structure of $R$. Therefore, let us forget about the coalgebraic structure of $R$ and write

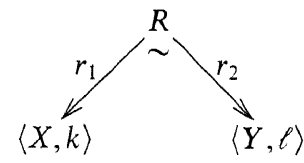

to express the fact that $\left\langle R, r_{1}, r_{2}\right\rangle$ is a span between the carriers $X$ and $Y$ that lifts to a bisimulation between the coalgebras $\langle X, k\rangle$ and $\langle Y, \ell\rangle$.

In the rest of this paper, with a slight abuse of notation, we often leave the legs $r_{1}$ and $r_{2}$ implicit and simply write $R$ for the whole span $\left\langle R, r_{1}, r_{2}\right\rangle$. Also, whenever possible, we use the infix notation $x R y$ to express the fact that there exists an element $z$ of $R$ such that $r_{1}(z)=x$ and $r_{2}(z)=y$. 
Proposition 4.1. (Coinductive extensions identify bisimilar elements) For every bisimulation $\left\langle R, r_{1}, r_{2}\right\rangle$ between two coalgebras $\langle X, k\rangle$ and $\langle Y, \ell\rangle$ the following diagram commutes.

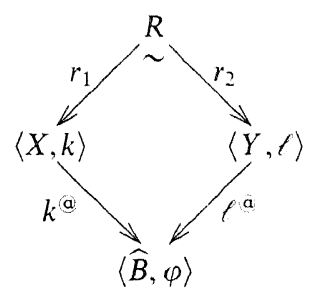

This is a trivial consequence of the fact that both composites in the diagram are coalgebra morphisms to the final coalgebra, hence they must be the same.

Semantically, this implies that if two programs are bisimilar, then they have the same final coalgebra semantics.

Corollary 4.1. (Strong extensionality) Every bisimulation on the final coalgebra has equal legs, that is, the following diagram commutes.

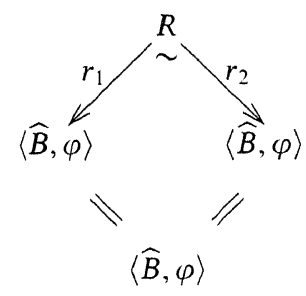

In other words, in a final coalgebra one cannot distinguish between bisimilar elements, which, semantically, amounts to the fact that final coalgebras of behaviours are internally fully abstract.

\subsection{Pullback bisimulations}

A canonical way of defining spans is by 'pullbacks'. Given two morphisms $f: X \rightarrow Z$ and $g: Y \rightarrow Z$ with a common codomain $Z$, their pullback is the universal among the spans between $X$ and $Y$ that form commuting squares with $f$ and $g$; that is, a $\operatorname{span}\langle P, p: P \rightarrow X, q: P \rightarrow Y\rangle$ such that $f \circ p=g \circ q$, and for every span $\left\langle P^{\prime}, p^{\prime}: P^{\prime} \rightarrow X, q^{\prime}: P^{\prime} \rightarrow Y\right\rangle$ such that $f \circ p^{\prime}=g \circ q^{\prime}$ there exists a unique morphism $u: P^{\prime} \rightarrow P$ such that $p^{\prime}=p \circ u$ and $q^{\prime}=q \circ u$. Diagrammatically:

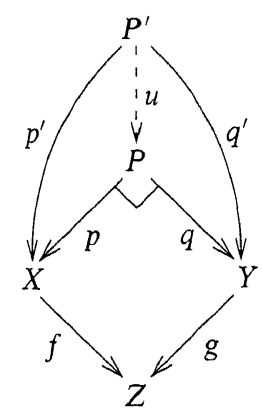


(Notice the notation for the pullback square.) In Set, the pullback of two functions $f$ and $g$ is the relation $R=\{\langle x, y\rangle \mid f x=g y\}$ with the evident projections as legs.

In a category $\underline{C}$ with pullbacks, one can define the internal equality $\operatorname{EQ}(X)$ of an object $X$ as the 'kernel pair' of the identity on $X$, that is, as the following pullback.

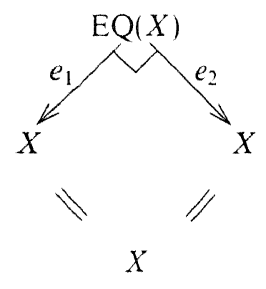

Proposition 4.2. For every coalgebra, the internal equality lifts to a coalgebraic bisimulation.

Proof. First, notice that the two legs $e_{1}$ and $e_{2}$ of the equality are the same. Next, consider the 'diagonal' $\mathrm{d}_{X}: X \rightarrow \mathrm{EQ}(X)$

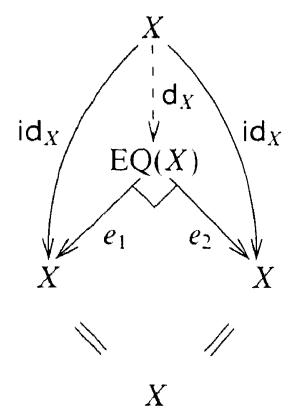

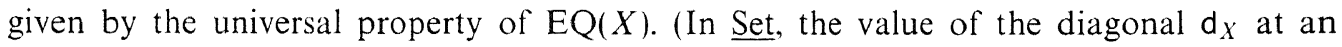
element $x$ of $X$ is the pair $\langle x, x\rangle$.) For any endofunctor $B$ and any $B$-coalgebra $\langle X, k\rangle$, since the composite $e_{i} \circ \mathrm{d}_{X}$ is the identity on $X$, the diagram

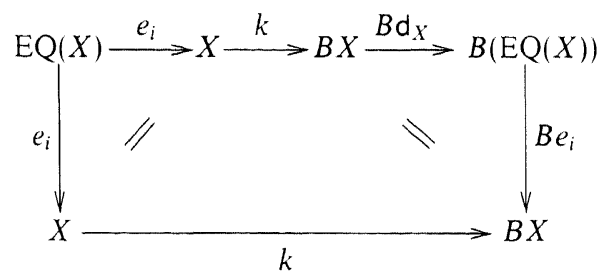

commutes. Hence, the composite $B \mathrm{~d}_{X} \circ k \circ e_{i}$ lifts the equality $\mathrm{EQ}(X)$ to a bisimulation on the coalgebra $\langle X, k\rangle$ :

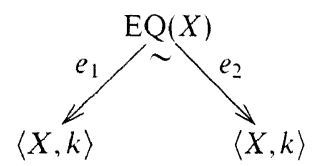


Consider the category $\underline{\operatorname{Bisim}}(B)$ having as objects spans lifting to bisimulations of an endofunctor $B$ and as morphisms triples of morphisms $\langle r, f, g\rangle$ making everything in sight in

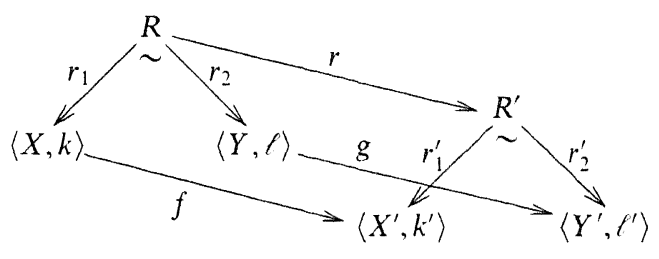

commute - where $f$ and $g$ are morphisms in $\underline{C}_{B}$, while $r$ is a morphism in $\underline{C}$. Then the equality $\operatorname{EQ}(\widehat{B})$ on (the carrier of) a final coalgebra is a final object in this category. This is an immediate consequence of the fact that $\operatorname{EQ}(\widehat{B})$ is a pullback (in $\underline{C}$ ):

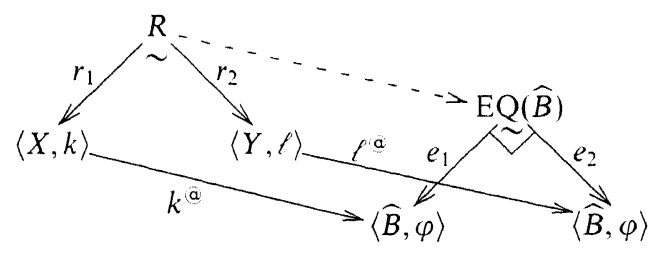

That is, from any span $R$ lifting to a bisimulation there is a mediating morphism to the equality $\mathrm{EQ}(\widehat{B})$ on a final coalgebra because the two legs of $R$ can be coinductively prolonged to form a suitable cone on (the carrier of) a final coalgebra. Therefore, we have the following proposition.

Proposition 4.3. The internal equality of the final coalgebra lifts to the final bisimulation.

This gives an alternative way of understanding the fact that coinductive extensions identify bisimilar elements.

\subsection{Coinduction pulled back to bisimulation}

If the pullback of the coinductive extensions of two coalgebras lifts to a bisimulation, as, for example, when the functor $B$ under consideration 'preserves weak pullbacks', then this pullback is the final bisimulation between the two coalgebras. In other words, coinduction can be 'pulled back' to bisimulation. Together with the above property that coinductive extensions identify bisimilar elements, this gives that two programs have the same final coalgebra semantics if and only if they are bisimilar.

Let $B$ be an endofunctor on a category $\underline{C}$ with pullbacks. Recall that pullbacks, like all universals, are determined by two conditions: uniqueness and existence. When only the existence part is known to hold one speaks of a weak pullback (and of a weak universal in general). Now, not all pullbacks lift to $B$-bisimulations, but a sufficient condition is that the endofunctor $B$ preserves weak pullbacks, that is, the image under $B$ of a weak pullback is still a weak pullback.

Proposition 4.4. If an endofunctor $B$ preserves weak pullbacks then pullbacks lift to $B$-bisimulations - $c f$. Aczel and Mendler (1989, Proposition 6.2). 
Proof. If the image under $B$ of a weak pullback is still a weak pullback, then every pullback in $\underline{C}$ of morphisms that are coalgebra homomorphisms lifts to a $B$ bisimulation. Indeed, since pullbacks are also weak pullbacks, for all $f:\langle X, k\rangle \rightarrow\langle Z, j\rangle$ and $g:\langle Y, \ell\rangle \rightarrow\langle Z, j\rangle$ in $\underline{C}_{B}$, the existence of a (possibly not unique) suitable coalgebra structure $\tilde{R}: R \rightarrow B R$ for the pullback $R$ of $f$ and $g$ in $\underline{C}$ is ensured by the weakly universal property of the weak pullback $B R$ :

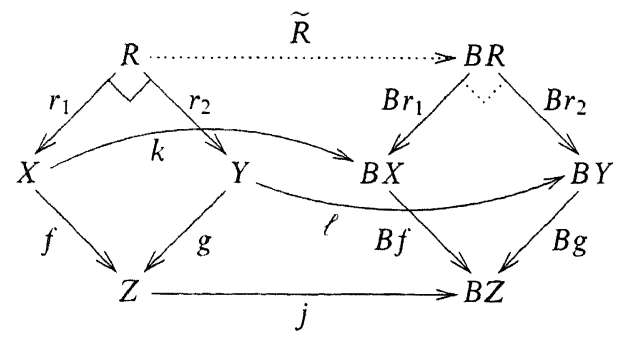

(The coalgebra structures $k$ and $\ell$ turn the legs of $R$ into a cone over the diagram for which $B R$ is a weak pullback.)

Let us check that the behaviour endofunctor $B X=\mathscr{P}(A c t \times X)$ preserves weak pullbacks and hence, by the above argument, pullbacks lift to (ordinary) bisimulations.

The problem of showing that the functor $B$ preserves weak pullbacks can be reduced to the problem of showing that $B$ maps (ordinary) pullbacks to weak pullbacks. Indeed, the following holds.

Observation 4.1. In Set, weak pullbacks embed pullbacks. That is, the diagram

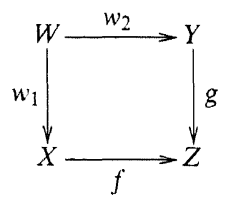

is a weak pullback diagram if and only if there exists an injection $m: R \gg W$ of the pullback $R=\{\langle x, y\rangle \mid f x=g y\}$ of $f$ and $g$ into $W$ such that

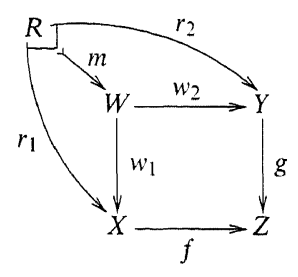

commutes.

Proposition 4.5. Pullbacks lift to ordinary bisimulations. From the above observation, if

$$
B R=\{\langle a, x, y\rangle \mid a \in \text { Act, } f x=g y\}
$$

is a weak pullback for $B f: B X \rightarrow B Z$ and $B g: B Y \rightarrow B Z$, the set $B W$ inherits the weak universality of $B R$ by means of the mediating morphism $B m: B R \rightarrow B W$. 
In turn, in order to prove that $B R$ is a weak pullback for $B f$ and $B g$ it suffices to prove that the (ordinary) pullback $R^{\prime}$ of $B f$ and $B g$ factorizes through it in the sense that there exists a function $h: R^{\prime} \rightarrow B R$ such that $r_{i}^{\prime}=B r_{i} \circ h$ :

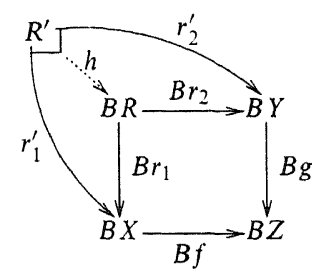

Indeed, then every other cone $\left(f^{\prime}, g^{\prime}\right)$ over the co-span $\langle B f, B g\rangle$ factorizes through the pullback as follows.

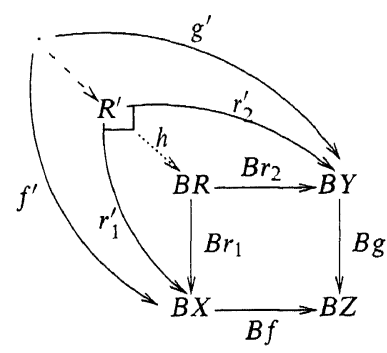

Let us now try to define such a function $h: R^{\prime} \rightarrow B R$ from the pullback $R^{\prime}$ of $B f$ and $B g$ to the image under $B$ of the pullback $R$ of $f$ and $g$. By definition of pullbacks in $\underline{\text { Set, the }}$ set $R^{\prime}$ consists of those pairs

$$
<\left\{<a_{i}, x_{i}>\right\}_{i \in I},\left\{<a_{j}, y_{j}>\right\}_{j \in J}>
$$

such that the index sets $I$ and $J$ are finite and

$$
B f\left\{<a_{i}, x_{i}>\right\}_{i \in I}=B g\left\{<a_{j}, y_{j}>\right\}_{j \in J} .
$$

The latter holds if and only if for every $i \in I$ there exists a $j \in J$ such that

$$
\left.\left.<a_{i}, f x_{i}\right\rangle=<a_{j}, g y_{j}>\quad \text { (that is, } a_{i}=a_{j}, f x_{i}=g y_{j}\right)
$$

and, conversely, for every $j \in J$ there exists an $i \in I$ such that $\left\langle a_{i}, f x_{i}\right\rangle=\left\langle a_{j}, g y_{j}\right\rangle$. But then one can define $h: R^{\prime} \rightarrow B R$ as mapping every pair

$$
\left\{<a_{i}, x_{i}>\right\}_{i \in I} R^{\prime}\left\{<a_{j}, y_{j}>\right\}_{j \in J}
$$

to the set

$$
\left\{<a_{i}, x_{i}, y_{j}>\mid a_{i}=a_{j}, f x_{i}=g y_{j}\right\} \in B R .
$$

This gives the desired factorization. Notice that the mediating function $h$ is not unique.

We have seen the category $\underline{\operatorname{Bisim}}(B)$ of all $B$-bisimulations. For every two $B$-coalgebras $\langle X, k\rangle$ and $\langle Y, \ell\rangle$, one can consider the evident subcategory $\underline{\operatorname{Bisim}}(\langle X, k\rangle,\langle Y, \ell\rangle)$ of $B$ bisimulations between $\langle X, k\rangle$ and $\langle Y, \ell\rangle$. 
Proposition 4.6. Let $B$ be an endofunctor on a category $\underline{C}$ with pullbacks and let the final $B$-coalgebra exist. If pullbacks lift to $B$-bisimulations, the pullback (in $\underline{C}$ ) of the coinductive extensions of two $B$-coalgebras $\langle X, k\rangle$ and $\langle Y, \ell\rangle$ lifts to the final object in $\underline{\operatorname{Bisim}}(\langle X, k\rangle,\langle Y, \ell\rangle)$.

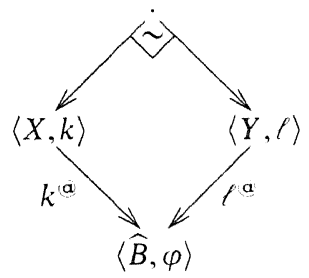

Write $\stackrel{k, t}{\sim}$ for the span obtained above by 'pulling back' the coinductive extensions of the coalgebra structures $k$ and $\ell$. Then, in Set, if the span $\stackrel{k, t}{\sim}$ lifts to a bisimulation,

$$
x \stackrel{k, f}{\sim} y \Longleftrightarrow k^{\mathrm{a}}(x)=\ell^{\mathrm{a}}(y)
$$

for any two elements $x \in X$ and $y \in Y$. (The implication from left to right follows the property that coinductive extensions always identify bisimilar elements.) Semantically, we have the following theorem.

Theorem 4.1. (Full abstraction) Given a coalgebraic operational model $\llbracket-\rrbracket:$ Prog $\rightarrow$ $B$ (Prog) of a language with programs $P \in$ Prog and behaviour $B$ such that pullbacks lift to $B$-bisimulations (as, for example, in Proposition 4.4), two programs $P, P^{\prime} \in$ Prog are $B$-bisimilar if and only if they have the same final coalgebra semantics. That is, for behaviours on 'concrete' categories like $\underline{\text { Set}}$,

$$
P \stackrel{\llbracket-\rrbracket}{\sim} P^{\prime} \Longleftrightarrow \llbracket P \rrbracket^{a}=\llbracket P^{\prime} \rrbracket^{a} .
$$

\subsection{From weakly final to final coalgebras}

Let us look at a concrete description in terms of trees and bisimulation of the final coalgebra for the finite power-set endofunctor $\mathscr{P}_{f i}$ on Set. Similar characterizations hold also for the power-set endofunctor $\mathscr{P}_{S}$ on SET and for the behaviours $B X=\mathscr{P}_{f}(A c t \times X)$ and $B X=\mathscr{P}_{S}($ Act $\times X)$.

Recall that the coalgebras of the finite power-set endofunctor $\mathscr{P}_{f i}$ are in a one-to-one correspondence with the finitely branching, directed graphs:

$$
x \longrightarrow x^{\prime} \Longleftrightarrow x^{\prime} \in k(x)
$$

Next, notice that the value of the coinductive extension of a coalgebra structure $k: X \rightarrow \mathscr{P}_{f}(X)$ at an element $x$ of $X$ is, omitting the final coalgebra isomorphism $\varphi: \widehat{\mathscr{P}}_{f} \cong \mathscr{P}_{f} \widehat{\mathscr{P}}_{f}$, given by the equation

$$
k^{(a}(x)=\left\{k^{(}\left(x_{i}\right) \mid x_{i} \in k(x)\right\},
$$


which can be seen as the recursive definition of a tree:

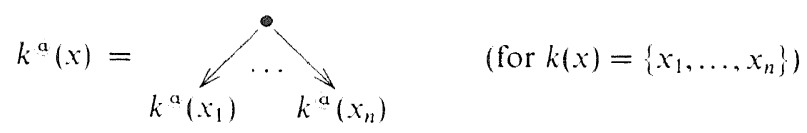

This is a rooted tree, finitely branching, and possibly of infinite depth. Neither nodes nor arcs are labelled. The set $\mathscr{T}$ of these rooted finitely branching trees can be seen as (the carrier of) a coalgebra of the finite power-set functor: every tree $\tau \in \mathscr{T}$ is mapped to the (finite) set $\left\{\tau_{1}, \ldots, \tau_{n}\right\}$ of children of its root:
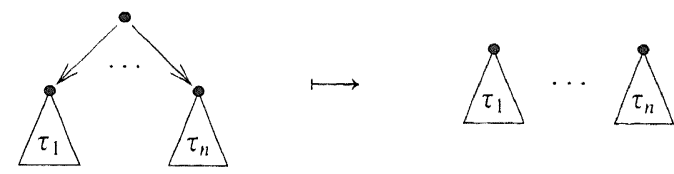

This coalgebra is not a final but a weakly final coalgebra, that is, it is a coalgebra that ensures the existence but not the uniqueness of coinductive extensions. For example, the coalgebra structure $k: X=\left\{x, x_{1}, x_{2}, x_{1}^{\prime}, x_{2}^{\prime}\right\} \rightarrow \mathscr{P}_{f i}(X)$

$$
k(x)=\left\{x_{1}, x_{2}\right\} \quad k\left(x_{1}\right)=\left\{x_{1}^{\prime}\right\} \quad k\left(x_{2}\right)=\left\{x_{2}^{\prime}\right\} \quad k\left(x_{1}^{\prime}\right)=0=k\left(x_{2}^{\prime}\right)
$$

can be extended to both the following trees.
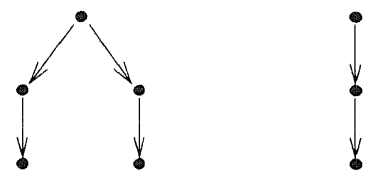

Proposition 4.7. The final coalgebra of the finite power-set functor is the set of rooted finitely branching trees quotiented by the corresponding (final) coalgebraic bisimulation.

In order to prove the above proposition, consider the quotient of a $\mathscr{P}_{f i}$-coalgebra $\langle X, k\rangle$ modulo its final bisimulation $R_{k}$ (which, by the above considerations, exists). Categorically, this amounts to taking the coequalizer $q: X \rightarrow X / R_{k}$ of the two legs $r_{1}, r_{2}: R_{k} \rightarrow X$ of the bisimulation $R_{k}$ :

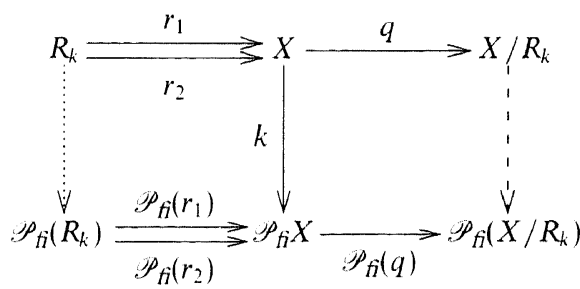

Notice this lifts to a coequalizer in the category of coalgebras. The coalgebra structure for $X / R_{k}$ is given by the universal property of the coequalizer. Indeed, since the legs of the bisimulation $R_{k}$ are coalgebra morphisms, the composite function $\mathscr{P}_{f i}(q) \circ k: X \rightarrow$ $\mathscr{P}_{f i}\left(X / R_{k}\right)$ equates the two legs of $R_{k}$. The corresponding unique mediating function from $X / R_{k}$ to $\mathscr{P}_{f i}\left(X / R_{k}\right)$ is the desired structure. Write $\langle X, k\rangle / R_{k}$ for this quotient coalgebra. 
Lemma 4.1. From every coalgebra there is at most one morphism to the quotient coalgebra $\langle X, k\rangle / R_{k}$.

Proof. Consider two coalgebra morphisms $f, g:\langle Y, \ell\rangle \rightarrow\langle X, k\rangle / R_{k}$. Since, as shown in the previous section, the internal equality always lifts to a coalgebraic bisimulation

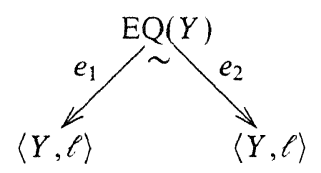

one has that the equality on $Y$ with as legs the composites $f \circ e_{1}, g \circ e_{2}: \operatorname{EQ}(Y) \rightarrow X / R_{k}$ lifts to a bisimulation on the quotient coalgebra $\langle X, k\rangle / R_{k}$ :

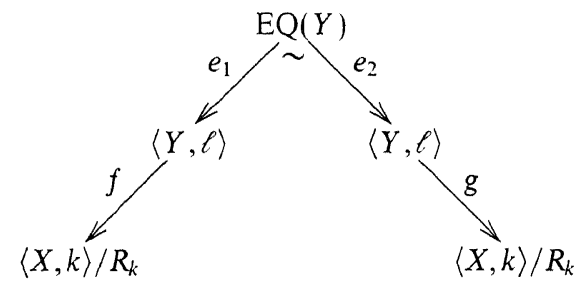

Therefore, for every $y \in Y, f(y)$ is bisimilar to $g(y)$. Since, by construction, the quotient $\langle X, k\rangle / R_{k}$ is strongly extensional, that is, bisimulation is the equality, one has that $f(y)$ is equal to $g(y)$ for every $y \in Y$, hence $f=g$ and the lemma is proved $-c f$. Aczel (1988, Theorem 2.19).

Therefore, the quotient modulo bisimulation of a weakly final $\mathscr{P}_{f^{-}}$-coalgebra is necessarily final: the existence of a morphism from every coalgebra is guaranteed by being the quotient of a weakly final coalgebra, the uniqueness is guaranteed by the above property of quotients modulo bisimulation. In particular, the weakly final coalgebra of rooted finitely branching trees can be thus quotiented by bisimulation to yield the final coalgebra of the finite power-set functor. This concludes the proof of the above proposition.

Notice that the finite power-set functor is not $\omega^{\mathrm{op}}$-continuous, that is, the limit of the following chain is not a fixed point for the finite power-set functor $\mathscr{P}_{f}$.

$$
1 \leftarrow 1
$$

Indeed, each object $\mathscr{P}_{f}{ }^{n} 1$ of the chain is the set of finitely branching trees with depth at most $n$, quotiented by bisimulation. Correspondingly, the following sequence of trees belongs to the above chain.

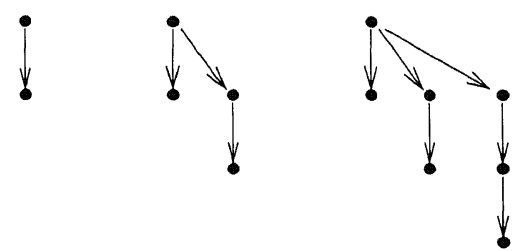


The problem is then that the limit has to contain the following tree with infinitely many branches,

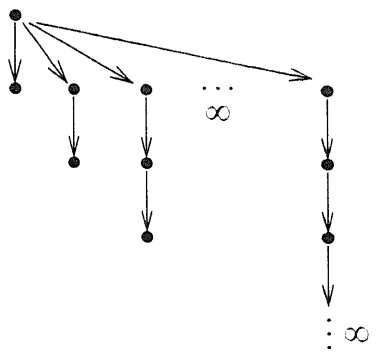

while the final coalgebra, as shown above, contains only finitely branching trees.

The above lemma can be used to prove the following generalization of Proposition 4.7.

Proposition 4.8. The quotient modulo $B$-bisimulation of a weakly final $B$-coalgebra, if it exists, is a final $B$-coalgebra.

\subsection{Bisimulations along morphisms}

The fact that coinductive extensions can be pulled back to bisimulations can be generalized to coinductive extensions along morphisms. This leads to a new, more general notion of ordinary bisimulation in which not only the actions but also some (properties of the) states can be observed.

Let $B: \underline{\mathrm{C}} \rightarrow \underline{\mathrm{C}}$ be an endofunctor such that the forgetful functor $U_{B}: \underline{\mathrm{C}}_{B} \rightarrow \underline{\mathrm{C}}$ has a right adjoint. Recall that final coalgebras $\widehat{B} \cong B \widehat{B}$ are a special case of cofree coalgebras $B_{\star} X$, namely $B_{\star} 1$, and that, correspondingly, the coinduction principle of final coalgebras generalizes to the arbitrary cofree coalgebras: for every coalgebra structure $k: Z \rightarrow B Z$ and morphism $f: Z \rightarrow X$, one has a unique coalgebra morphism $f^{b}:\langle Z, k\rangle \rightarrow B_{\star} X$, namely the coinductive extension of $k$ along $f-c f$. Section 3.2.

Next, consider a $B$-bisimulation

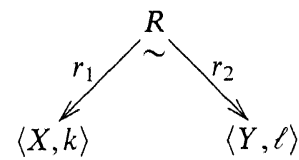

and two morphisms $f: X \rightarrow Z$ and $g: Y \rightarrow Z$ in $\underline{C}$ such that the diagram

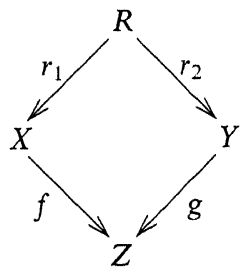


commutes. Then also the diagram

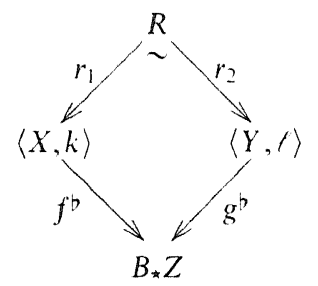

commutes, because both composites $f^{b} \circ r_{1}$ and $g^{b} \circ r_{2}$ fit as the unique coinductive extension of the (no matter which!) coalgebra structure on $R$ along the composite $f \circ r_{1}=g \circ r_{2}: R \rightarrow Z$.

If pullbacks lift to $B$-bisimulation, then the pullback (in the base category) of the coinductive extensions $f^{b}$ and $g^{\text {b }}$ of $k$ and $\ell$ along $f$ and $g$ is the final object in the evident category of bisimulations making (9) commute.

Example 4.1. Consider the simple behaviour $B X=1+$ Act $\times X$ (see Section 2.2) and, correspondingly, ordinary bisimulation for deterministic transition systems. Let the set Act of actions be trivial, that is, let Act be made of only one action $a$. Let $\langle X, k\rangle$ and $\langle Y, \ell\rangle$ be the same coalgebra having as carrier the set $\mathscr{Z}$ of integers and as structure $\ell: \mathscr{Z} \rightarrow B(\mathscr{Z})$ the one corresponding to the following (deterministic) transition system: 0 is inert, a positive integer $n$ performs a transition to its predecessor $n-1$, and a negative integer $-n$ performs a transition to its successor $-n+1$ :

$$
0 \downarrow * \quad n \stackrel{a}{\longrightarrow} n-1 \quad-n \stackrel{a}{\longrightarrow}-n+1 .
$$

Finally, let $Z$ be the three-elements set $\{0, \&, \diamond\}$. Thus:

$$
X=\mathscr{Z}=Y \quad Z=\{0, \leftrightarrow, \diamond\} \quad \text { Act }=\{a\} .
$$

Now, different bisimulations are possible according to the choice of the functions $f, g$ : $\mathscr{Z} \rightarrow\{0, \leftrightarrow, \diamond\}$. Let us fix the function $g: \mathscr{Z} \rightarrow\{0, \bullet, \diamond\}$ to be the one mapping odd numbers to and even numbers to $\diamond$. If $f$ is equal to $g$, then every number is bisimilar to itself and to its opposite. For instance,

$$
f^{b}(-3)=\propto \stackrel{a}{\longrightarrow} \diamond \stackrel{a}{\longrightarrow} \stackrel{a}{\longrightarrow} 0=g^{b}(3)
$$

and thus -3 is bisimilar to 3 (with respect to $g$ ).

The above amounts to assuming that one can observe in both transition systems whether a number is odd or even. If, instead, in the first transition system one can observe this only for positive numbers, thus, eg, $f(-n)=0$ and $f(n)=g(n)$, then one has that a positive number $n$ is bisimilar to both $-n$ and $n$ (with respect to $f$ and $g$ ) but its opposite $-n$ is not bisimilar to any number in the second transition system.

Finally, if one cannot observe at all in the first transition system whether a number is odd or even (that is, $f(z)=0$ for all $z \in \mathscr{Z}$ ), then only the two 0's are bisimilar.

(Notice that the morphisms $f$ and $g$ can be regarded as abstract interpretations of the states.) 
Example 4.2. Another example is when one has a distinguished subset $\operatorname{Obs}(X) \subset X$ of states that are 'observable'. This can be expressed by taking $Z=\operatorname{Obs}(X) \cup\{\perp\}$ and $f: X \rightarrow \operatorname{Obs}(X) \cup\{\perp\}$ to be

$$
f(x)= \begin{cases}x & \text { if } x \in \operatorname{Obs}(X) \\ \perp & \text { otherwise }\end{cases}
$$

Notes. The notion of an ordinary bisimulation stems from the work of Park (1981) and Milner (1980) on concurrency. Coalgebraic bisimulations for relations between sets were introduced in Aczel and Mendler (1989). (See also the dual algebraic congruences in Manes (1976, page 167).) The more general use of spans stems from Joyal et al. (1993), where, however, 'open morphisms' are used instead of coalgebraic homomorphisms. The idea is that, given a category $\underline{M}$ of operational models, and a subcategory $\underline{P} \hookrightarrow \underline{M}$ of computations (or 'path objects'), two models $X$ and $Y$ (that is, two objects of $\underline{M}$ ) are $\underline{P}$-bisimilar if there is a span of $\underline{P}$-open morphisms in $\underline{M}$. When $\underline{M}$ is the category of transition systems and $\underline{\mathrm{P}}$ is a suitable category of 'abstract' finite transition sequences,

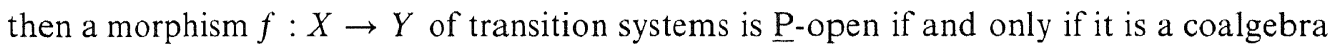
morphism $f:\langle X, k\rangle \rightarrow\langle Y, \ell\rangle$, for $k$ and $\ell$ the coalgebra structures corresponding, via (1), to the transition systems on $X$ and $Y$, respectively. It would be interesting to investigate whether this correspondence between open morphisms and coalgebra homomorphisms holds also for different notions of path objects and endofunctors.

For a large class of functors over cpos, a generalized notion of bisimulation is considered in Pitts (1994a), which, more subtly, amounts to first lifting the behaviour endofunctors to a category of relations and then studying the coalgebras therein. A systematic way of defining these liftings for 'simple' functors is described in Hermida and Jacobs (1995).

\section{Non-well-founded sets}

This section is devoted to a coalgebraic presentation of Peter Aczel's theory of 'non-wellfounded sets' (Aczel 1988). A categorical duality is proved between the 'anti-foundation axiom' giving non-well-founded sets and the 'foundation axiom': it is shown that the former is equivalent to postulating that 'the universe $V=\mathscr{P}_{S} V$ is a final coalgebra', while the latter is equivalent to ' $V=\mathscr{P}_{S} V$ is an initial algebra'. (The endofunctor $\mathscr{P}_{S}$ maps a class to the class of its (small) subsets.)

The semantic motivation for the use of anti-foundation is that it permits one to prove the 'Special Final Coalgebra Theorem' (Aczel 1988), which states that, under mild assumptions, the greatest fixed point of an endofunctor on (possibly non-well-founded) sets is a final coalgebra.

The Special Final Coalgebra Theorem is stated in terms of the 'Solution Lemma'. The final coalgebra presentation of anti-foundation adopted here renders this lemma (and its equivalence with anti-foundation) trivial. Correspondingly, the 'uniformity on maps' condition that an endofunctor has to satisfy in order for the Special Final Coalgebra Theorem to hold can be formulated in a more transparent way than in Aczel (1988). 


\subsection{Basic set theory}

One way of understanding the abstract notion of a set is as a collection $x$ such that its elements have 'no internal structure whatsoever' and $x$ itself has 'no internal structure except for equality and inequality of pairs of elements' - $c f$. Lawvere (1976, page 119). Axiomatically, this corresponds to taking the membership relation ' $\epsilon$ ' as the only primitive notion of set theory and to postulating the following 'extensionality axiom', the first axiom of set theory.

Extensionality: Two sets are equal iff they have the same elements.

Next, for every property $P$ in a (first-order) language with membership and equality only, one would like the collection $\{x \mid \mathrm{P}(x)\}$ of sets that have the property $\mathrm{P}$ to be a set. However, Russell's paradoxical set $\{x \mid x \notin x\}$ shows that this 'strong comprehension axiom' cannot be stated in its full generality. One needs to consider properties relative to the elements of an already defined set. This leads to the 'comprehension axiom', the second axiom of set theory.

Comprehension: For every property $\mathrm{P}$ and every set $v$, the collection

$$
\{x \mid \mathrm{P}(x) \wedge x \in v\}
$$

is a set.

As comprehension can be applied only to members of already defined sets, it is necessary to postulate the existence of some sets, either primitive or derived by applying some basic operators, as follows.

Empty Set: There exists a set 0 with no elements.

Pairing, Union, Power-Set: $\{x, y\}, \bigcup x, \mathscr{P}(x)$ are all sets, for $x, y$ sets.

As usual, $\bigcup x$ and $\mathscr{P}(x)$ stand for the collection of all members of members of $x$ and the collection of all subsets of $x$, respectively. In turn, the subset relation ' $\subseteq$ ' can be derived from the membership relation:

$$
x \subseteq y \Longleftrightarrow \forall v(v \in x \Rightarrow v \in y)
$$

By means of the union operator, one can define an operator $s$ acting as successor as follows: $s(x)=x \cup\{x\}$. The existence of an infinite set can be stated by postulating the existence of a set containing the natural numbers, as follows.

Infinity: There exists a set containing 0 and closed under the successor operator $s$.

(The axioms above, as well as those given later in this paper, are written for convenience in natural language but note that they can also be expressed in the language of set theory - see, for example, Levy (1979). 
Further useful notions can be derived from the above axioms, like, for instance, the notion of ordered pair :

$$
<x, y>=\{x,\{x, y\}\} .
$$

A formal definition of function can then be given as a collection $f$ of ordered pairs such that for every $x$ there exists a unique $y$ with $\langle x, y\rangle \in f$. The following two additional axioms about functions are then usually added.

Replacement: The image of a set under a function is a set.

Choice: Every surjective function has a 'right inverse'.

A right inverse for a function $f: a \rightarrow b$ is a function $g: b \rightarrow a$ such that $f \circ g$ is the identity on $b$. The above axiom of choice is equivalent to postulating that for every set $a$ there exists a choice function, that is, a function $f$ such that, for every $x \in a, f(x) \in x$.

The above axioms (extensionality, comprehension, empty set, pairing, union, power set, infinity, replacement, choice) are the basic axioms of set theory; let us call the theory associated with (that is, the collection of all sentences derivable from) them basic set theory and the corresponding category of sets and functions Set. (Basic set theory is usually called $Z F C^{-}$in the literature - see, for example, Levy (1979).)

Classes. Even though the collection $\{x \mid \mathrm{P}(x)\}$ of all sets $x$ having a given property $\mathrm{P}$ might not be a set, it can still be of interest for set theory. Such 'specifiable' collections are called classes. Clearly, a set is a class, but the converse is not true, in which case one speaks of a proper class. (Also the terminology 'large set', vs 'small set', is used.) In the rest of this paper, lower case letters are used for (small) sets and capital letters for classes.

The equality between classes is determined by their small elements. That is, two classes $X=\{x \mid \mathrm{P}(x)\}$ and $Y=\left\{x \mid \mathrm{P}^{\prime}(x)\right\}$ are equal if and only if $\mathrm{P}$ and $\mathrm{P}^{\prime}$ hold for the same (small) sets.

An example of a proper class is the universe of sets, namely the collection of all sets:

$$
V=\{x \mid x=x\} \text {. }
$$

(Since the property $x=x$ trivially holds for all sets, the class $V$ is the collection of all sets indeed.) Notice that different properties may specify the same class. For instance, any property other than ' $x=x$ ' that holds for all sets can be used to specify the universe.

Next, let SET be the category of classes and (class) functions corresponding to basic set theory. The claim is that the universe $V$ can be seen as the carrier of both an algebra and a coalgebra structure of the endofunctor

$$
\mathscr{P}_{S}: \underline{\mathrm{SET}} \rightarrow \underline{\mathrm{SET}} \quad X \mapsto\{x \mid x \text { is a set } \wedge x \subseteq X\}
$$

that maps a class to the class of its (small) subsets. By taking subsets rather than subclasses one overcomes the cardinality problem, which prevents the unrestricted power-set from having a fixed-point.

Proposition 5.1. The universe $V$ is a strict fixed point $V=\mathscr{P}_{S} V$ of the endofunctor $\mathscr{P}_{S}: \underline{\mathrm{SET}} \rightarrow \underline{\mathrm{SET}}$. 
Proof. The universe $V$ is, by definition, the largest class, hence, since $\mathscr{P}_{S} V$ is itself a class, $\mathscr{P}_{S} V \subseteq V$. For the converse it is sufficient to prove that every set $x$ is a subset of $V$. That is, for every $y \in x, y$ is also in $V$. This is immediate from the fact that $y$ is a set.

Therefore, the identity on $V$ can be seen both as a $\mathscr{P}_{S}$-algebra and as a $\mathscr{P}_{S}$-coalgebra structure for $V$.

\subsection{Well-founded sets and foundation}

From the axioms of basic set theory alone it is not possible to draw a canonical picture of what the universe looks like, a picture independent of the specific interpretation one might give to the theory. This was already felt as a problem in the early developments of set theory. The solution was found in the 'foundation axiom', which was then added to basic set theory. This axiom restricts the universe to the 'smallest' of all possible ones. Then the picture arises of a universe in which sets are constructed by iterative applications of the power-set operator starting from the empty set. Every set has a rank, namely the stage at which it appears in such a 'cumulative hierarchy'.

In this section it is proved that the foundation axiom is equivalent to postulating that the universe $V=\mathscr{P}_{S} V$ is the initial algebra of the power-set endofunctor $\mathscr{P}_{S}$ on SET.

A set $x$ is well-founded with respect to the membership relation ' $\epsilon$ ' if either it is empty or has a least element with respect to $\epsilon$. In other words, there is no infinitely descending chain of elements starting from $x$. Correspondingly, let the class

$$
W=\{x \mid x \text { is well-founded with respect to the relation } \in\}
$$

be the universe of well-founded sets.

The 'foundation axiom' amounts to postulating that all sets in the universe $V$ are well-founded.

\section{Foundation Axiom:
\[ V=W . \]}

Now, notice that the class $\mathscr{P}_{S} W$ of (small) subsets of well-founded sets is the same as $W$, because the elements of a well-founded set are themselves well-founded. Thus

$$
\not_{S} W=W
$$

and the identity on $W$ can be seen as a $\mathscr{P}_{S}$-algebra structure.

Proposition 5.2. The universe of well-founded sets is an initial $\mathscr{P}_{S}$-algebra.

Proof. For every $\mathscr{P}_{S}$-algebra structure $h: \mathscr{P}_{S} X \rightarrow X$ there exists a unique function $h^{*}: W \rightarrow X$ such that the following diagram commutes.

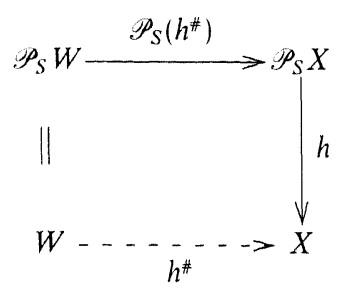


That is,

$$
\begin{aligned}
& h^{*}(0)=h(0) \\
& h^{*}\left\{x_{i}\right\}_{I}=h\left\{h^{*}\left(x_{i}\right)\right\}_{I} .
\end{aligned}
$$

The proof is by straightforward induction on the (well-founded!) membership relation $\in$.

An immediate consequence of the initiality of $W$ is the existence of a 'rank' function, mapping every well-founded set to a suitable 'ordinal'. An ordinal is a well-founded set that is totally ordered by the membership relation and is 'transitive'. (A transitive set is a set $x$ such that every element $y \in x$ is also a subset $y \subseteq x$.) Correspondingly, one can form the class $\mathcal{O}$ of all ordinals, which is a subclass of $W$.

If $\alpha$ and $\beta$ are two ordinals such that $\beta \in \alpha$, one usually writes $\beta<\alpha$. The first ordinals are: $0, s(0), s^{2}(0)$, and so on. The first limit ordinal is $\omega=\bigcup_{n \in N} s^{n}(0)$, which, by the infinity axiom, is indeed a set. In general, because every ordinal is totally ordered by $\in$, the union $\bigcup\left\{\alpha_{i}\right\}_{I}$ of a set $\left\{\alpha_{i}\right\}_{I}$ of ordinals is the least upper bound of the $\alpha_{i}$ 's. As a consequence, the union operator is a $\mathscr{P}_{S}$-algebra structure on the class of ordinals:

$$
\bigcup: \mathscr{P}_{S}(\mathbb{C}) \rightarrow \mathbb{C} \quad\left\{\alpha_{i}\right\}_{I} \mapsto \bigcup\left\{\alpha_{i}\right\}_{I} .
$$

The inductive extension rank $=\bigcup^{\#}: W \rightarrow \mathscr{O}$ of this algebra structure on $\mathcal{O}$ is the function assigning a 'rank' to every well-founded set. This can be thought of as the stage at which a well-founded set is constructed in an idealized construction starting from the empty set and then iteratively applying the power-set functor $\mathscr{P}_{S}$ :

$$
\begin{aligned}
& \operatorname{rank}(0)=0 \\
& \operatorname{rank}\left\{x_{i}\right\}_{I}=\bigcup\left\{\operatorname{rank}\left(x_{i}\right)\right\}_{I} .
\end{aligned}
$$

Another consequence of the initiality of $W$ is that $W=\mathscr{P}_{S} W$ is the least (pre-) fixed point for $\mathscr{P}_{S}$ :

$$
W=\operatorname{lfp}\left[\mathscr{P}_{S}\right]
$$

That is, for every class $X$ such that $\mathscr{P}_{S} X \subseteq X$, one has that $W \subseteq X$. Indeed, regarding the inclusion of $\mathscr{P}_{S} X$ into $X$ as a function $\kappa: \mathscr{P}_{S} X \hookrightarrow X$, one has that its inductive extension $\kappa^{*}: W \rightarrow X$ is of the following form:

$$
\begin{aligned}
& \kappa^{*}(0)=0 \\
& \kappa^{\#}\left\{x_{i}\right\}_{I}=\kappa\left\{\kappa^{\#}\left(x_{i}\right)\right\}_{I} .
\end{aligned}
$$

Then, to see that $k^{\#}$ is the inclusion of $W$ into $X$, it suffices to notice that the power-set functor

\section{$\mathscr{P}_{S}$ 'preserves inclusion functions'}

that is, if $l: X \hookrightarrow Y$ is the inclusion of a subclass $X$ of $Y$ into $Y$, then the function $\mathscr{P}_{S}(l): \mathscr{P}_{S} X \rightarrow \mathscr{P}_{S} Y$ is the inclusion of $\mathscr{P}_{S} X$ into $\mathscr{P}_{S} Y$.

Usually, initial algebras are unique up to isomorphism, but in this setting one has a stronger result:

$$
\mathscr{P}_{S} X=X \text { is an initial } \mathscr{P}_{S} \text {-algebra } \Longleftrightarrow X=W \text {. }
$$


That is, any other initial algebra that is a (strict) fixed point of $\mathscr{P}_{S}$ is not only isomorphic but equal to $W$. In order to prove this, that is, the non-trivial implication from left to right, one can use very much the same argument as the one used above to prove that $W$ is the least fixed point of $\mathscr{P}_{S}$.

Therefore, by taking the $X$ in $(10)$ to be the universe $V$, one has that the foundation axiom ' $V=W$ ' is equivalent to postulating that the universe $V$ is an initial algebra of the power-set functor.

Theorem 5.1. (Foundation is initiality)

$$
V=W \Longleftrightarrow \mathscr{P}_{S} V=V \text { is an initial } \mathscr{P}_{S} \text {-algebra. }
$$

\subsection{Anti-foundation and finality}

As shown in the Introduction, not all sets occurring in mathematical practice are wellfounded. In order to ensure the existence of non-well-founded sets, one can postulate the 'anti-foundation axiom', which here is shown to be the dual of the initial algebra formulation of 'foundation':

$$
\begin{array}{ll}
\text { Foundation: } & \mathscr{P}_{S} V=V \text { is an initial } \mathscr{P}_{S} \text {-algebra. } \\
\text { Anti-Foundation: } & V=\mathscr{P}_{S} V \text { is a final } \mathscr{P}_{S} \text {-coalgebra. }
\end{array}
$$

That is, anti-foundation postulates that the universe is the 'largest' possible one, while foundation postulates that it is the 'smallest'.

Theorem 5.2. (Final $\mathscr{P}_{S}$-Coalgebra Theorem.) The endofunctor

$$
\mathscr{P}_{S}: \underline{\text { SET }} \rightarrow \underline{\text { SET }} \quad X \mapsto\{x \mid x \text { is a set } \wedge x \subseteq X\}
$$

on the category SET of classes (that is, large sets) that are definable within basic set theory, has a final coalgebra.

The above theorem stating the existence of a final $\mathscr{P}_{S}$-coalgebra is an instance of the "final coalgebra theorem' in Aczel and Mendler (1989). It can also be seen as an instance of Theorem 3.1, replacing the finite power-set $\mathscr{P}_{f i}$ by $\mathscr{P}_{S}$. Correspondingly, the core of the proof is the fact that the coalgebras of the restriction $\mathscr{P}: \underline{\text { Set }} \rightarrow \underline{\text { Set }}$ of the endofunctor $\mathscr{P}_{S}$ to the category $\underline{\text { Set }}$ of small sets form a 'generating class' for the $\mathscr{P}_{S}$-coalgebras; that is, every $\mathscr{P}_{S}$-coalgebra can be obtained by quotienting the disjoint union of a suitable class of $\mathscr{P}$-coalgebras.

By considering the unlabelled version of the correspondence (1) given in Section 2, one has that there is a one-to-one correspondence between directed (small) graphs $\langle X, \rightarrow\rangle$ and coalgebras $k: X \rightarrow \mathscr{P} X$ : for every $x, x^{\prime} \in X$,

$$
x \longrightarrow x^{\prime} \Longleftrightarrow x^{\prime} \in k(x) .
$$

Similarly, the coalgebras of the power-set functor $\mathscr{P}_{S}$ are the same as the directed locally small' graphs, that is, the (possibly large) directed graphs such that the collection of children of every node is a (small) set.

Peter Aczel's original formulation of the anti-foundation axiom is in terms of small graphs and 'decorations'. A decoration for (the graph corresponding to) a $\mathscr{P}_{S}$-coalgebra 
$\langle X, k\rangle$ is a coalgebra morphism from $\langle X, k\rangle$ to $V=\mathscr{P}_{S} V$

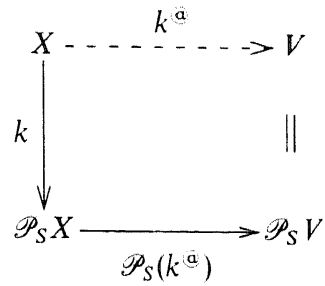

That is, a function $f$ from $X$ to the universe $V$ such that, for every $x \in X$,

$$
f(x)=\left\{f\left(x^{\prime}\right) \mid x^{\prime} \in k(x)\right\} .
$$

In terms of graphs, this corresponds to a function mapping every node to a set as follows:

$$
f(x)=\left\{f\left(x^{\prime}\right) \mid x \longrightarrow x^{\prime}\right\} .
$$

Therefore, by definition of final coalgebra, the coalgebra $V=\mathscr{P}_{S} V$ is final if and only if every (directed) locally small graph has a unique decoration. Now, the claim is that 'locally small' can be replaced by 'small' in the above equivalence.

Lemma 5.1. Every locally small graph has a unique decoration if (and only if) every small graph has a unique decoration.

Proof. The proof is by contradiction. Assume that every small graph has a unique decoration and that there are two distinct decorations $f$ and $g$ of (a coalgebra $\langle X, k\rangle$ corresponding to) a locally small graph. Then there is a node $x \in X$ such that

$$
f(x) \neq g(x) .
$$

Now, the subgraph of $\langle X, k\rangle$ accessible from $x$ is not only locally small but also (totally) small, that is, there are only set-many nodes accessible from $x$, because every node has only set-many children. But then $f$ and $g$ are both decorations for this small subgraph, which, by hypothesis, implies that

$$
f(x)=g(x) .
$$

(The same argument can be used to prove that the class of small $\mathscr{P}_{S}$-coalgebras forms a generating class for the $\mathscr{P}_{S}$-coalgebras.)

As a consequence, the postulate ' $V=\mathscr{P}_{S} V$ is a final $\mathscr{P}_{S}$-coalgebra' is equivalent to Peter Aczel's original formulation of anti-foundation (which is equivalent to Forti and Honsell's ' $X_{1}$-axiom' (Forti and Honsell 1983)).

Anti-Foundation Axiom: Every directed small graph has a unique decoration.

So we have the following theoerem.

Theorem 5.3. (Anti-Foundation is finality) Every directed small graph has a unique decoration if and only if $V=\mathscr{P}_{S} V$ is a final $\mathscr{P}_{S}$-coalgebra.

Notice that no axiom is needed in order to obtain a unique decoration for a well-founded graph: one can check that the class WG of well-founded directed small graphs is a (strict) fixed point for the power-set functor $\mathscr{P}_{S}$, and, moreover, that $\mathscr{P}_{S}(W G)=W G$ is an initial 
$\mathscr{P}_{S}$-algebra. Therefore $W G$ is isomorphic to the universe of well-founded sets $W$ and the image under this isomorphism of a well-founded graph is its unique decoration - $c f$. 'Mostowski's collapsing lemma' in Aczel (1988).

When anti-foundation is postulated, non-well-founded graphs also have a unique decoration, but the converse is not true anymore. That is, there exist (non-well-founded) sets that 'decorate' different graphs. An example is the archetypal non-well-founded set, namely the self-singleton set

$$
\Omega=\{\Omega\}
$$

that is a member (and the only member) of itself. If anti-foundation is assumed, then both the root of the graph with one node and one arc

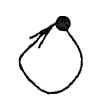

and the root of the graph consisting in one infinite path

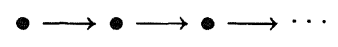

are necessarily mapped to $\Omega$ by the corresponding unique decorations.

Notice that, besides applications in the semantics of programming languages (for example, Aczel (1988), Mukai (1991), Rutten and Turi (1993), Aczel (1994), Baldamus (1994), Honsell and Lenisa (1995) and Hartonas (1997)), non-well-founded sets have been extensively used in Situation Theory (for example, Barwise and Etchemendy (1987)), where they are better known as hypersets. (Correspondingly, models of the universe of non-wellfounded sets are also called hyperuniverses.)

Reasoning about non-well-founded sets: bisimulation. By the extensionality axiom, the equality between two sets is determined by the membership relation. One of the consequences of foundation is that, since then the membership relation is well-founded, one can use induction to reason about (the equality between) sets. Categorically, this induction principle follows from the fact that foundation postulates that the universe is an initial algebra. Dually, anti-foundation, by postulating that the universe is a final coalgebra, gives a coinduction principle for reasoning about (possibly non-well-founded) sets.

Now, as shown in Section 4, if an endofunctor preserves weak pullbacks, coinduction (with respect to its final coalgebra) can be 'pulled back' to the corresponding coalgebraic notion of bisimulation. In particular, the power-set functor $\mathscr{P}_{S}$ does preserve weak pullbacks; the proof is essentially the same as the one given in Section 4 for the behaviour $B X=\mathscr{P}($ Act $\times X)$.

By instantiating the general definition of coalgebraic bisimulation (Section 4 ) to the $\mathscr{P}_{S}$-coalgebras, one has that a $\mathscr{P}_{S}$-bisimulation for a coalgebra $\langle X, k\rangle$ is a span $R$ such that, for all $x_{1}, x_{2}$ in $X$, if $x_{1} R x_{2}$, then

- if $x_{1} \longrightarrow x_{1}^{\prime}$, then $x_{2} \longrightarrow x_{2}^{\prime}$ for some $x_{2}^{\prime}$ such that $x_{1}^{\prime} R x_{2}^{\prime}$

- and, conversely, if $x_{2} \longrightarrow x_{2}^{\prime}$, then $x_{1} \longrightarrow x_{1}^{\prime}$ for some $x_{1}^{\prime}$ such that $x_{1}^{\prime} R x_{2}^{\prime}$. 
(Here the notation $x \longrightarrow x^{\prime}$ stands for there is an arc from $x$ to $x^{\prime}$ in the graph corresponding to the coalgebra $\langle X, k\rangle$ '.)

In particular, a $\mathscr{P}_{S}$-bisimulation $R$ for the universe $V$ is such that, for every set $x$ and $y$, if $x R y$, then for every set $x^{\prime}$ in $x$ there exists a set $y^{\prime}$ in $y$ such that $x^{\prime} R y^{\prime}$ and, conversely, for every set $y^{\prime}$ in $y$ there exists a set $x^{\prime}$ in $x$ such that $x^{\prime} R y^{\prime}$. Therefore, by strong extensionality,

$$
x=y \Longleftrightarrow \exists R\left(x R y \wedge\left(\forall x^{\prime} \in x, \exists y^{\prime} \in y, x^{\prime} R y^{\prime}\right) \wedge\left(\forall y^{\prime} \in y, \exists x^{\prime} \in x, x^{\prime} R y^{\prime}\right)\right) .
$$

\subsection{Systems of set-equations as coalgebras}

The self-singleton non-well-founded set $\Omega=\{\Omega\}$ can be seen as the unique solution of the 'set-equation'

$$
x=\{x\}
$$

In general, all non-well-founded sets arise from systems of set-equations with, on the left-hand side, variables $x \in X$, and, on the right-hand side, well-founded sets, possibly containing variables from $X$. This is the content of the 'Solution Lemma', which is proved below in an elementary way thanks to the coalgebraic account of anti-foundation (and the initial algebra presentation of well-founded sets).

The definition of the universe of well-founded sets $W$ can be made parametric: for every (possibly large) set $X$, the expanded universe of well-founded sets $W X$ is the class of all well-founded sets with variable $x \in X$. That is, every set in $W X$ is either empty, or an element of $X$, or it has a least element with respect to the membership relation $\in$. For $X=0$ this yields the standard universe $W 0$ of well-founded sets. Thus, in the rest of this paper, $W$ stands for an operator mapping a (large) set to the corresponding expanded universe of well-founded sets, rather than for the simple universe of well-founded sets.

The fact that $W 0$ is the least (strict) fixed point of the power-set functor $\mathscr{P}_{S}$ and that $\mathscr{P}_{S} W 0=W 0$ is an initial $\mathscr{P}_{S}$-algebra generalizes as follows: the class $W X$ is the least (strict) fixed point of the endofunctor $X+\mathscr{P}_{S}(-)$ on $\underline{\mathrm{SET}}$ and

$$
X+\mathscr{P}_{S} W X=W X
$$

is an initial algebra for this endofunctor. The universal property given by initiality can be used to extend the operator $W$ to a functor, like in Diagram (3) of Section 3.1. That is, for every function $f: X \rightarrow Y$, the function $W f: W X \rightarrow W Y$ is the inductive extension of the algebra structure $\operatorname{inr}_{Y}: \mathscr{P}_{S} W Y \rightarrow W Y$ along the composite $\eta_{Y} \circ f: X \rightarrow W Y$, where the left injection $\eta_{Y}=\operatorname{inl}_{Y}: Y \rightarrow W Y$ is the usual insertion-of-variables function.

Observation 5.1. By the above definition and the definition of free algebras in Section 3.2, the forgetful functor from the category of $\mathscr{P}_{S}$-algebras to SET has a left adjoint, namely the functor mapping a class $X$ to the free algebra (11).

(In general, every free $\mathscr{P}_{S}$-algebra over a possibly large set $X$ can be used to model the universe of Zermelo-Fraenkel set theory expanded with elements of $X$ as atoms. This fact can be seen as an instance of a more general result in Joyal and Moerdijk (1995), 
namely Theorem 1155 , which is stated in terms of free 'Zermelo-Fraenkel algebras' and intuttomatic set theory

Now, the wea in that a system of "set-equations" like, for example,

$$
\begin{aligned}
& x=\{x, y\}\} \\
& y=\{y, 0\}
\end{aligned}
$$

can he seen as a function $k$ mapping the variables $x, y, \ldots \in X$ of the system to elements

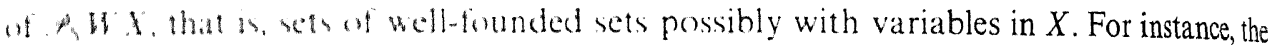
ahowe whem correypunds w a function $k:\left\{x, y=X \rightarrow \mathscr{P}_{S} W X\right.$ mapping $x$ to $\{x,\{y\}$ and $1, w, 4$. Therefore, in general, a system of set-equations in $X$ is a coalgebra $\langle X, k\rangle$ of the compente colufunctur PS on SET.

In order to solve a system of set-equations $\langle X, k\rangle$, one can (postulate anti-foundation and use the thallity of the universe $l=P_{S} V$. For this, one first needs to extend the

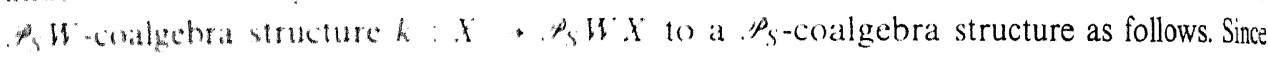
$W A-X . A X$ a coproduct, one can form the copair $[k, i d]: W X \rightarrow \mathscr{P}_{S} W X$ of $k$ and the dentuly id on,$W X$. This is a $P_{S}$-coalgebra structure behaving as $k$ on $x \in X$ and as the dentry on .,$W X$. Its conductive extension $\bar{k}=[k, \text { id }]^{\text {a }}: W X \rightarrow V$ with

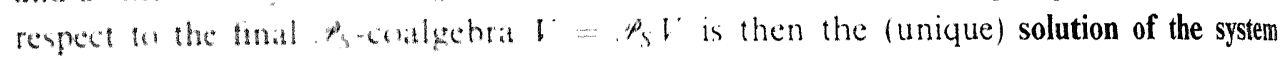
$k: X * W \cdot$ of set-equations:

$$
\begin{aligned}
& 1 \eta_{k}=w x^{k-[k, i d]^{\prime \prime}=1} \\
& \text { h. } \quad[h, i d]
\end{aligned}
$$

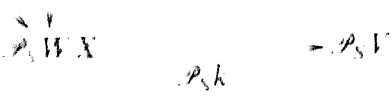

()mutung, as ustal, the mectums, and letting $r$ and $r^{\prime}$ range over objects of type $\mathscr{P}_{S} W$. one has that

$$
h(x), k(n), h w \quad \text { and } k(v)=\left\{k\left(v^{\prime}\right) \mid v^{\prime} \in v\right\} \text {. }
$$

for example, the unque solution of equation $k(x)=\{x\}$ is the self-singleton (non-wellfounded set $h(x), k(w)$, that is, $k(x)=\Omega$. Similarly, the solution of the above system 1121 is

$$
\begin{aligned}
& k(x)=k(x), k(y) i j \\
& k(y)=k(y), 0) .
\end{aligned}
$$

In terms of graphs, the wets $k 1, t$ and $k(y)$ correspond to

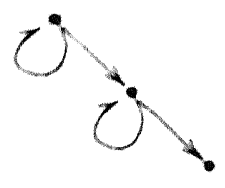

and

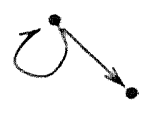

respectively. 
The Solution Lemma. The above property that every system of set-equations has a unique solution, is called the Solution Lemma in Aczel (1988) - see also Barwise and Etchemendy (1987, Chapter 3). It is obtained assuming the anti-foundation axiom. Conversely, postulating the Solution Lemma, one can prove that $V=\mathscr{P}_{S} V$ is the final $\mathscr{P}_{S}$-coalgebra. Indeed, for every $\mathscr{P}_{S}$-coalgebra $\langle X, k\rangle$, one obtains

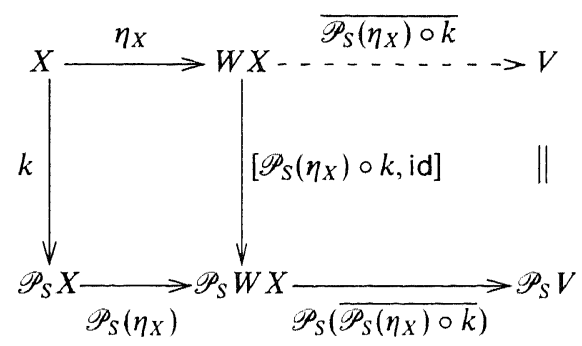

The desired coinductive extension of the coalgebra structure $k: X \rightarrow \mathscr{P}_{S} X$ is given by the composite coalgebra morphism

$$
k^{@}=\overline{\mathscr{P}_{S}\left(\eta_{X}\right) \circ k} \circ \eta_{X}: X \rightarrow V
$$

Therefore we have the following proposition.

Proposition 5.3. The Solution Lemma is equivalent to anti-foundation.

Notice that, assuming anti-foundation, the upper rectangle in the following diagram commutes, because all other sub-diagrams commute.

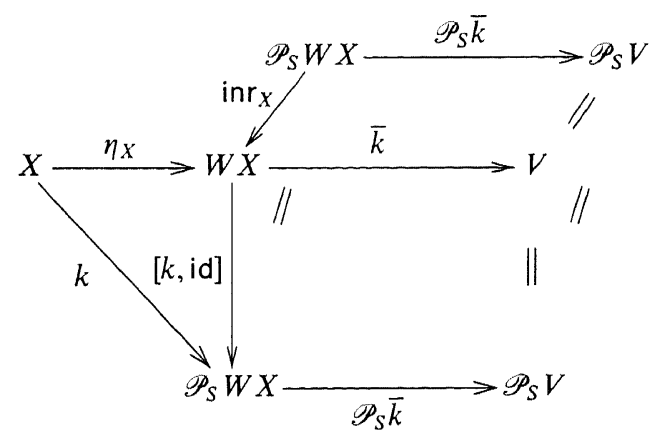

Therefore, the solution $\bar{k}: W X \rightarrow V$ of a system of set equations $\langle X, k\rangle$ is not only a $\mathscr{P}_{S}$-coalgebra morphism but also a $\mathscr{P}_{S}$-algebra morphism from $\left\langle W X, \operatorname{inr}_{X}\right\rangle$ to $\mathscr{P}_{S} V=V$. The algebra $\left\langle W X, \operatorname{inr}_{X}\right\rangle$ is a free $\mathscr{P}_{S}$-algebra over $X$.

The Substitution Lemma from freeness. In the present approach, the proof of the Solution Lemma is trivial. The original proof, instead, makes use of a substitution lemma (Aczel 1988). This lemma asserts that, for every function $f: X \rightarrow V$, there exists a unique extension $f^{\sharp}: W X \rightarrow V$ of $f$ to $W X=X+\mathscr{P}_{S} W X$ such that, omitting the injections,

$$
f^{\sharp}(x)=f(x) \quad \text { and } \quad f^{\sharp}(v)=\left\{f^{\sharp}\left(v^{\prime}\right) \mid v^{\prime} \in v\right\} .
$$


Now, this also becomes trivial here, because of the initial algebra presentation of the expanded universe of well-founded sets $W X$. Indeed, the desired function $f^{\#}: W X \rightarrow V$ is the inductive extension of the $\mathscr{P}_{S}$-algebra structure $\mathscr{P}_{S} V=V$ along $f: X \rightarrow V$. That is,

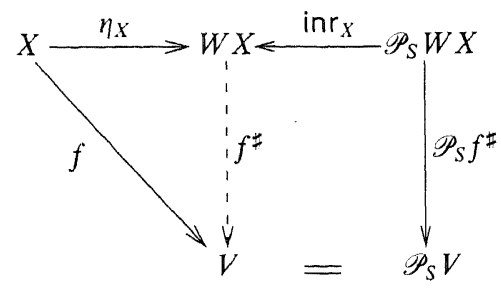

Notice that, in contrast with Aczel (1988), anti-foundation is not used here.

\subsection{From greatest fixed points to final coalgebras}

The greatest (strict) fixed point $V=\mathscr{P}_{S} V$ of the power-set functor $\mathscr{P}_{S}$ can be seen as the final coalgebra of the restriction of the functor $\mathscr{P}_{S}$ to the subcategory $\underline{\mathbf{S E T}}_{\subset}$ of inclusion functions. Anti-foundation postulates that this final coalgebra lifts to a final coalgebra in SET. If an endofunctor is 'uniform on maps', then, assuming anti-foundation, its final coalgebra in the subcategory $\underline{\mathrm{SET}}_{c}$ also lifts to a final coalgebra in $\underline{\mathrm{SET}}$. This is the content of the 'Special Final Coalgebra Theorem'.

In this section, a new formalization of the notion of uniformity on maps in terms of natural transformations is given. The proof of the theorem is then rephrased in terms of this definition.

Let $F$ be an endofunctor on SET. A post-fixed point $X \subseteq F X$ for $F$ can be seen as an inclusion function $X \hookrightarrow F X$, and hence as an $F$-coalgebra structure on $X$. If the endofunctor $F$ preserves inclusion functions, that is, $F$ applied to $X \hookrightarrow Y$ is an inclusion $F X \hookrightarrow F Y$, then one can restrict $F$ to the subcategory $\underline{\mathrm{SET}}_{\subset}$ of classes and inclusion functions. The post-fixed points of $F$ are then its coalgebras in this subcategory. In particular, the final $F$-coalgebra in $\underline{\mathrm{SET}}_{\odot}$, if it exists, is the greatest (post-)fixed point

$$
g f p[F]=F(g f p[F])
$$

of $F$. The claim is that if $F$ is 'uniform on maps', then, assuming anti-foundation, $g f p[F]=F(g f p[F])$ is also a final coalgebra.

Intuitively, an endofunctor on SET is uniform on maps if it is completely determined by its action on objects (that is, classes). Most endofunctors are thus uniform on maps. For instance, consider the endofunctor $X \mapsto A \times X$ mapping a class $X$ to its product with a fixed class $A$. Given a function $f: X \rightarrow Y$, the value of $A \times f$ at an element $\langle a, x\rangle$ of $A \times X$ is the pair $<a, f(x)>\in A \times Y$ that is obtained by applying $f$ to the $x \in X$ in $A \times X$. This suggests that the class $X$ should be regarded as a class of variables and that, in general, the action of a functor $F$ uniform on maps on a function $f$ should simply be the substitution of the variables $x$ occurring in $F X$ by $f(x)$. 
Formally, this can be expressed by means of the expanded universe of well-founded sets $W X=X+\mathscr{P}_{S} W X$. What one needs is a natural transformation

$$
\rho: F \Rightarrow \mathscr{P}_{S} W
$$

that, for every $X$, 'embeds' $F X$ into $\mathscr{P}_{S} W X$ - the class of sets of (well-founded) sets having $x \in X$ as variables.

Naturality amounts to having, for every function $f: X \rightarrow Y$, the following diagram commute:

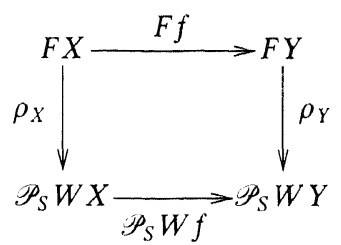

It should be an 'embedding' in the sense that, for every $X$ and for every $v \in F X$, by 'forgetting' the distinction between variables and sets in $\rho_{X}(v) \in \mathscr{P}_{S} W X$ one should get back the original set $v$. This operation of forgetting the distinction between variables and sets in objects of type $\mathscr{P}_{S} W$ can be made formal as follows.

Consider the inductive extension $\varepsilon_{V}: W V \rightarrow V$ of the $\mathscr{P}_{S}$-algebra structure $\mathscr{P}_{S} V=V$ along the identity on $V$ :

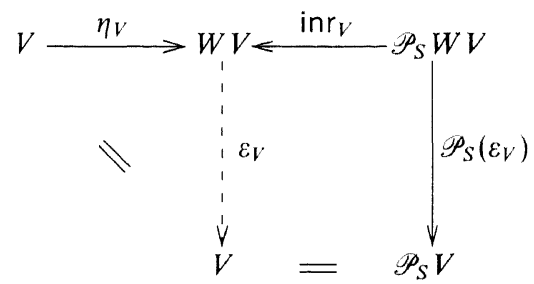

Omitting, as usual, the injections, one has, for every $v \in W V$, that $\varepsilon_{V}(v)=v$ if $v$ is a variable and $\varepsilon_{V}(v)=\left\{\varepsilon_{V}\left(v_{i}\right)\right\}_{I}$ if $v=\left\{v_{i}\right\}_{I}$.

Definition 5.1. (Uniformity on maps) An endofunctor $F: \underline{\mathrm{SET}} \rightarrow \underline{\mathrm{SET}}$ is uniform on maps if there exists a natural transformation

$$
\rho: F \Rightarrow \mathscr{P}_{S} W
$$

such that

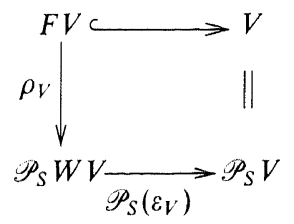

commutes.

Before setting out to prove the Special Final Coalgebra Theorem, notice that, by Observation 5.1 and the definition of adjunction, there is a bijection (natural in $X$ and $\langle Y, h\rangle$ ) between functions $f: X \rightarrow Y$ and $\mathscr{P}_{S}$-algebra morphisms $g:\langle W X$, inr $X\rangle \rightarrow\langle Y, h\rangle$. 
This bijection maps $f$ to its left adjunct

$$
f^{\#}=\varepsilon_{\langle Y, h\rangle} \circ W f,
$$

and $g$ to its right adjunct

$$
g^{b}=U g \circ \eta_{X}=g \circ \eta_{X}
$$

where $\varepsilon_{\langle Y, h\rangle}: W Y \rightarrow Y$ (the 'counit' of the adjunction) is the inductive extension of the right injection $\operatorname{inr}_{Y}: \mathscr{P}_{S} W Y \rightarrow W Y$ along the identity on $Y$.

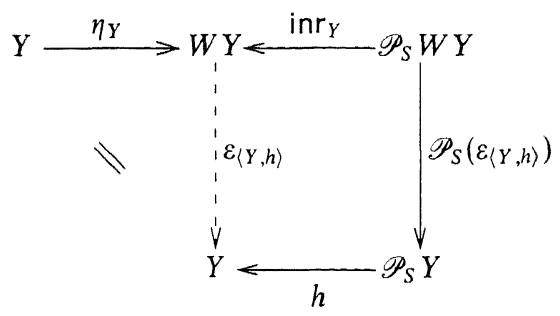

(Thus, in particular, the above function $\varepsilon_{V}: W V \rightarrow V$ is the value of the counit at the algebra $\mathscr{P}_{S} V=V$. Formally, $\varepsilon_{V}=U \varepsilon_{\left(\mathscr{P}_{S} V=V\right)}=\varepsilon_{\left(\mathscr{P}_{S} V=V\right)}$, where $U$ is the forgetful functor mapping algebras to their carriers.)

Theorem 5.4. (The Special Final Coalgebra Theorem) Let $F$ be an endofunctor on SET that cuts down to an endofunctor on the subcategory SET $_{c}$ of inclusion functions.

If $F$ is uniform on maps, then, assuming anti-foundation, its final coalgebra

$$
g f p[F]=F(g f p[F])
$$

in $\underline{\mathrm{SET}}_{\subset}$ lifts to a final $F$-coalgebra in $\underline{\mathrm{SET}}$.

Proof. Consider an $F$-coalgebra structure

$$
k: X \rightarrow F X \text {. }
$$

By uniformity on maps, there exists a function $\rho_{X}: F X \rightarrow \mathscr{P}_{S} W X$, hence $k$ can be made into a system of set-equations in $X$ by composing it with $\rho_{X}$. Take its solution $\overline{\rho_{X} \circ k}: W X \rightarrow V$ and define a function $f$ from $X$ to $V$ as the right adjunct (13) of this solution with respect to the above adjunction; that is,

$$
f=\left(\overline{\rho_{X} \circ k}\right)^{b}=\overline{\rho_{X} \circ k} \circ \eta_{X}: X \rightarrow V .
$$

Diagrammatically:

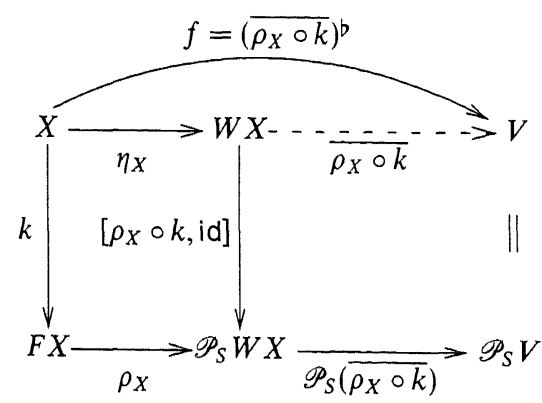


The claim is that, under the above hypotheses, $f$ is an $F$-coalgebra morphism from $\langle X, k\rangle$ to $g f p[F]=F(g f p[F])$, that is, the diagram

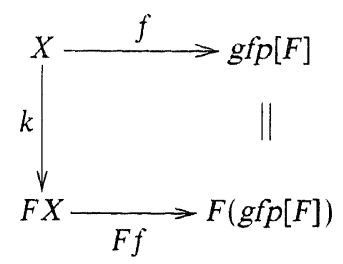

commutes. More precisely: let $Y$ be the image under $f$ of $X$. The function $f: X \rightarrow V$ can be factorized, like every function in SET, as

$$
X \stackrel{f}{\rightarrow} Y \hookrightarrow V .
$$

The claim is then that the class $Y$ is a post-fixed point for $F$, that is, $Y \subseteq F Y$, and $f$ is a coalgebra morphism from $\langle X, k\rangle$ to $Y \hookrightarrow F Y$, that is,

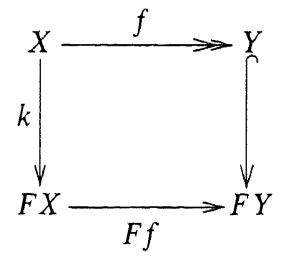

commutes.

If the above holds, since $F$ cuts down to an endofunctor on the subcategory $\underline{S E T}_{c}$ of inclusions, the composition of $f$ the inclusion $Y \hookrightarrow g f p[F]$ of $Y$ into the greatest fixed point of $F$ is an $F$-coalgebra morphism:

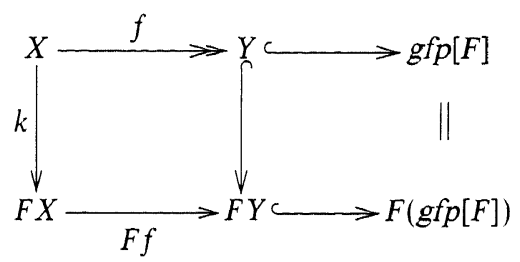

In order to prove the above claim, notice that everything in sight in the following diagram commutes:

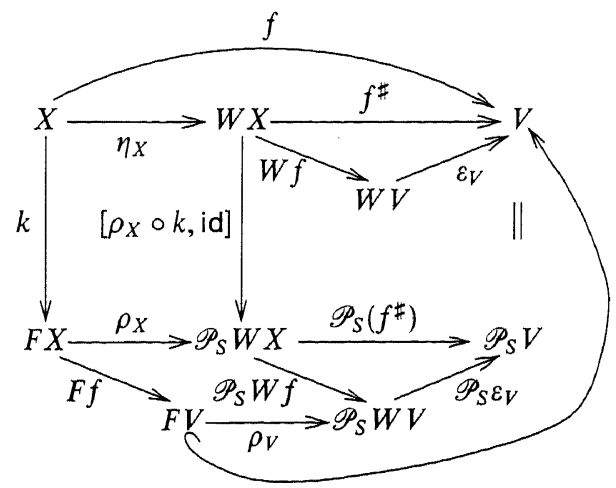


In particular, the outer diagram does commute, hence

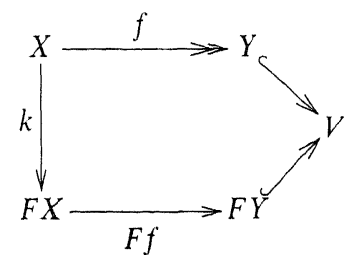

Therefore, for all $x \in X$,

$$
f(x)=(F f \circ k)(x)
$$

which implies that the image $Y$ of $X$ under $f$ is included in the image of $F X$ under $F f$, and hence

$$
Y \subseteq F Y
$$

and $f$ is a coalgebra morphism from $\langle X, k\rangle$ to $Y \hookrightarrow F Y$.

Therefore, for every $F$-coalgebra $\langle X, k\rangle$, there exists a coalgebra morphism to $g f p[F]=$ $F(g f p[F])$. Moreover, this morphism is unique. Indeed, the above arguments also show that every coalgebra morphism from $\langle X, k\rangle$ to $g f p[F]=F(g f p[F])$ fits as the right adjunct $\left(\overline{\rho_{X} \circ k}\right)^{b}$ of the unique solution of a system of set-equations, hence it is unique.

Notes. An alternative (but more restrictive) form of the Special Final Coalgebra Theorem in the standard category of ordinary sets is presented in Paulson (1995).

The Special Final Coalgebra Theorem is the 'dual' of the standard fact that least (strict) fixed points of most endofunctors on SET are initial algebras - cf. Aczel (1988, Theorem 7.6). It gives an elementary way of finding final coalgebras, at the price of assuming anti-foundation. For instance, under foundation, the endofunctor $B X=A c t \times X$ has the empty set 0 as the unique strict fixed point, while, under anti-foundation, the empty set is the least fixed point and the set $A c t^{(t)}$ of infinite words over the alphabet $A c t$ is the greatest fixed point of $B$ : the Special Final Coalgebra Theorem then says that $A c t^{(t)}=A c t \times A c t^{(\nu}$ is a final $B$-coalgebra.

Notice that one can prove the (non-strict!) fixed point $A c t^{(1)} \cong A c t \times A c t^{(1)}$ is a final $B$-coalgebra in Set, independently of the use of anti-foundation. In general, as shown in Aczel and Mendler (1989), endofunctors to which the Special Final Coalgebra Theorem apply always have a final coalgebra in the category of ordinary (possibly large) sets. Thus, unless one is really interested in strict fixed points $\widehat{B}=B \widehat{B}$, rather than fixed points up to isomorphism $\widehat{B} \cong B \widehat{B}$, the interest can be shifted from non-well-founded sets and greatest fixed points to ordinary sets and final coalgebras.

\section{Partial orders}

When interpreting the programs of a language, one would like, in general, not only to establish whether two programs $P, P^{\prime}$ are behaviourally equivalent, but also when one program $P^{\prime}$ is (behaviourally) 'better' than another program $P$. Write $P \lesssim P^{\prime}$ for this relation, with the intended meaning that $P^{\prime}$ can simulate everything $P$ does, but not 
necessarily vice versa. This 'simulation' relation ' $\leqslant$ ' should be reflexive (that is, $P \leqslant P$ ) and transitive (that is, if $P \leqslant P^{\prime}$ and $P^{\prime} \leqslant P^{\prime \prime}$, then $P \leqslant P^{\prime \prime}$ ), that is, it should be a preorder for the programs.

The main contribution of this section is a full abstraction theorem with respect to this behavioural preorder. This is obtained by working in an 'order-enriched' setting where the morphism sets are partially ordered and composition yields order-preserving maps.

\subsection{Order-enriched categories and final coalgebras}

A preorder $\leqslant$ for a set $X$ is a partial order if it is anti-symmetric in the sense that if $x \leqslant y$ and $y \leqslant x$ then $x=y$, for all $x, y \in X$. The category Poset is the category of partially ordered sets (posets) $\langle X, \leqslant\rangle$ and monotone functions $f:\langle X, \leqslant\rangle \rightarrow\left\langle X^{\prime}, \leqslant^{\prime}\right\rangle$, that is, order-preserving functions $f: X \rightarrow X^{\prime}$. To ease the notation, we will write $X$ for a poset $\langle X, \leqslant\rangle$ and $\leqslant x$ for its order. Also, whenever possible, we will drop the subscript and write simply $\leqslant$ for $\leqslant X$.

For every two posets $X, Y$, the set $\underline{\operatorname{Poset}}(X, Y)$ of monotone functions $f: X \rightarrow Y$ from $X$ to $Y$ is partially ordered pointwise:

$$
f \leqslant g \Longleftrightarrow f(x) \leqslant_{Y} g(x) \text { for all } x \in X .
$$

That is, the 'hom-sets' of Poset are objects of Poset itself. Moreover, for every two hom-sets $\underline{P o s e t}(X, Y)$ and $\underline{\operatorname{Poset}}(Y, Z)$, the composition function

$$
\circ: \underline{\operatorname{Poset}}(Y, Z) \times \underline{\operatorname{Poset}}(X, Y) \rightarrow \underline{\operatorname{Poset}}(X, Y)
$$

is monotone

$$
g \leqslant g^{\prime}, f \leqslant f^{\prime} \Rightarrow g \circ f \leqslant g^{\prime} \circ f^{\prime} .
$$

That is, composition is a morphism of Poset.

Loosely speaking, a category $\underline{\mathrm{C}}$ whose hom-sets are objects of a category $\underline{\mathrm{V}}$ and whose composition, for every $X, Y, Z$ in $\underline{\mathrm{C}}$, is a morphism

$$
\circ: \underline{\mathrm{C}}(Y, Z) \times \underline{\mathrm{C}}(X, Y) \rightarrow \underline{\mathrm{C}}(X, Z)
$$

of $\underline{V}$ is called a $\underline{V}$-enriched category or, for short, a $\underline{V}$-category; when $\underline{C}=\underline{V}$, then $\underline{C}$ is also called self-enriched. Notice the assumption that $\underline{\mathrm{V}}$ has binary products $\times$. In general, this assumption can be relaxed: it is sufficient to assume that $\underline{\mathrm{V}}$ has a 'monoidal' structure. (For more on enriched categories see Lawvere (1973), Kelly (1982) and Casley et al. (1991).)

Thus Poset is a self-enriched category. Another example is the category $\mathbf{C p o}$ of complete partial orders (cpos), that is, partial orders closed under least upper bounds of $\omega$-chains, and continuous functions, that is, (monotone) functions that preserve these least upper bounds.

A functor between two $\underline{V}$-enriched categories is a $\mathbf{V}$-functor if it is 'locally' a morphism of $\underline{\mathrm{V}}$, that is, the action of $F$ on each hom-set is a morphism of $\underline{\mathrm{V}}$. Thus, a Poset-functor $F$ is a locally monotone functor, that is,

$$
f \leqslant g \Rightarrow F f \leqslant F g
$$


and a Cpo-functor is a locally continuous functor $F$, that is, a functor such that

$$
F\left(\bigsqcup_{I} f_{i}\right)=\bigsqcup_{I} F\left(f_{i}\right)
$$

for every $\omega$-chain $\left\{f_{i}\right\}_{1}$ of continuous functions. Every Cpo-functor is also a Poset-functor.

Initial algebras (and final coalgebras) of Cpo-endofunctors can be obtained by means of the (dual of the) following colimit construction.

Lemma 6.1. (Basic Lemma (Smyth and Plotkin 1982)) Let $F$ be an endofunctor on a category $\underline{C}$ with an initial object 0 . Let $\Delta$ be the diagram

$$
0 \stackrel{0_{F 0}}{\longrightarrow} F 0 \stackrel{F 0_{F 0}}{\longrightarrow} F^{2} 0 \stackrel{F^{2} O_{F 0}}{\longrightarrow} \cdots
$$

obtained by the iterative application of the functor $F$ to the unique morphism $0_{F 0}: 0 \rightarrow F 0$ given by initiality. If $\Delta$ has a colimit and $F$ preserves it, that is,

$$
F(\operatorname{Colim}(\Delta)) \cong \operatorname{Colim}(F \Delta),
$$

then $\operatorname{Colim}(\Delta)$ is the carrier of an initial algebra for $F$. Its initial algebra structure is obtained by first noticing that the colimiting cocone for $\Delta$ (without the first morphism) is a cocone for $F \Delta$, and then taking the unique mediating morphism from $F(\operatorname{Colim}(\Delta))$ to $\operatorname{Colim}(\Delta)$.

The dual of this lemma is obtained by reversing the morphisms and replacing initial by final, colimit by limit, and algebra by coalgebra.

In Smyth and Plotkin (1982) it is shown that every Cpo-functor on a Cpo-category satisfies the hypotheses of the basic lemma, hence it has an initial algebra. The proof involves the use of the category $\mathrm{Cpo}^{E}$ of 'embedding-projection pairs', that is, pairs of morphisms in opposite directions satisfying suitable conditions. The use of this auxiliary category also gives a tool for treating mixed variant-contravariant functors $F$ over Cpo, like the function space of type $\mathrm{Cpo}^{\mathrm{op}} \times \underline{\mathrm{Cpo}} \rightarrow \underline{\mathrm{Cpo}}$, as covariant functors $F^{E}$ over $\underline{\mathrm{Cpo}}$.

If $F$ is a Cpo-endofunctor, then the corresponding endofunctor $F^{E}$ on $\underline{C p o}^{E}$ satisfies the hypotheses of the basic lemma and of its dual. Hence it has both an initial algebra and a final coalgebra; moreover, the former is the initial algebra for the original endofunctor $F$ on $C$ po as well. However, the final coalgebra of $F^{E}$ is not the final $F$-coalgebra; for this, one has to move to the category pCpo of cpos and partial continuous functions - every Cpo-endofunctor $F$ on pCpo has both an initial algebra and a final coalgebra, namely the initial algebra and the final coalgebra for the corresponding endofunctor $F^{E}$ on $\underline{\mathrm{Cpo}}^{E}=\underline{\mathrm{pCpo}}^{E}$.

The 'coalgebraic completeness' of pCpo makes of it a good candidate for the orderenriched version of the full abstraction theorem, but we find it easier to work with a category of total morphisms that is isomorphic to $\underline{\mathrm{pCpo}}$, namely the category $\mathbf{C p p o}_{\perp}$ of pointed cpos (that is, cpos with a least element ' $\perp$ ') and strict continuous functions (that is, functions that also preserve the least element). (The above considerations on the construction of initial algebras and final coalgebras in $\mathrm{pCpo}$ remain valid in the isomorphic category $\underline{\mathrm{Cppo}}_{\perp}$.)

When an endofunctor $F$ has both an initial algebra $\psi: F \bar{F} \cong F$ and a final coalgebra $\varphi: F \cong F \widehat{F}$, there is a canonical morphism from the carrier $\bar{F}$ of the initial algebra 
to the carrier $\widehat{F}$ of the final coalgebra, namely the coinductive extension of the inverse $\psi^{-1}: \bar{F} \cong F \bar{F}$ of the initial algebra isomorphism. If this canonical morphism is itself an isomorphism, the initial algebra and the final coalgebra are canonically isomorphic.

Categories in which all endofunctors have both an initial algebra and a final coalgebra and, moreover, are canonically isomorphic are called algebraically compact in Freyd (1992). An example of such a category is $\underline{C p p o}_{\perp}$, when regarded as a Cpo-category. This can be proved by means of the 'limit-colimit coincidence' of categories of embedding-projection pairs (Smyth and Plotkin 1982, Theorem 2). In particular, the one-element set $\{\perp\}$ (with trivial order) is a null object in $\underline{C p p o}_{\perp}$ (and in the corresponding category of embeddingprojections pairs), that is, it is both an initial and a final object. Correspondingly, the diagram (14) whose colimit is an initial algebra can be turned into a diagram with the same objects whose limit is a final coalgebra by simply reversing the morphisms.

To summarize, we have the following theorem.

Theorem 6.1. (Algebraic compactness) Every Cpo-endofunctor on $\underline{C p p o}_{\perp}$ has both an initial algebra and a final coalgebra, and, moreover, they are canonically isomorphic.

The final coalgebras of Cpo-endofunctors can be characterized as the 'maximally final' ones. The definition of maximal finality requires the notion of a 'lax coalgebra morphism' from enriched category theory (Kelly 1982), which, for the order-enrichment, cuts down to the following notion. Let $F: \underline{\text { Poset }} \rightarrow$ Poset be an endofunctor on Poset and $\langle X, k\rangle$ and $\left\langle X^{\prime}, k^{\prime}\right\rangle$ be two $F$-coalgebras. Then a lax coalgebra morphism $f:\langle X, k\rangle \rightarrow\left\langle X^{\prime}, k^{\prime}\right\rangle$ is a monotone function $f: X \rightarrow X^{\prime}$ such that

$$
F f \circ k \geqslant k^{\prime} \circ f \text {. }
$$

Diagrammatically:

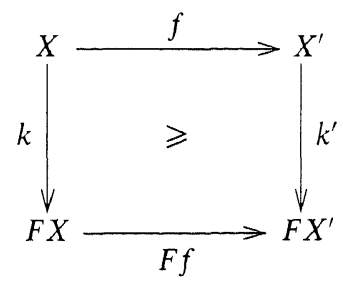

Thus lax coalgebra morphisms generalize (strict) coalgebra morphisms.

A coalgebra of an endofunctor $F$ on a Poset-category is maximally final if it is final and, for every $F$-coalgebra $\langle X, k\rangle$, the unique coinductive extension $k^{\text {a }}$ of $k$ is maximal among all lax morphisms from $\langle X, k\rangle$ to the final $F$-coalgebra. That is, if $\varphi: \widehat{F} \cong F \widehat{F}$,

$$
F f \circ k \geqslant \varphi \circ f \Rightarrow k^{@} \geqslant f .
$$

Proposition 6.1. Final coalgebras of locally continuous endofunctors are maximally final.

Proof. See Fiore (1996b, Proposition 6.7).

\subsection{Ordered bisimulations}

The order-enriched generalization of the notion of coalgebraic bisimulation should ensure that $(a)$ the inequality relation on a final coalgebra lifts to a coalgebraic ordered 
bisimulation and that $(b)$ the inequality relation on a maximal final coalgebra should be maximally-final in the category of coalgebraic ordered bisimulations. This motivates the following definition from Fiore (1996b), improving a previous definition in Rutten and Turi (1993).

Definition 6.1. (Coalgebraic ordered bisimulation) Let $\langle X, k\rangle$ and $\langle Y, \ell\rangle$ be coalgebras of an endofunctor $B$ on a Poset-category. A span $\left\langle R, r_{1}: R \rightarrow X, r_{2}: R \rightarrow Y\right\rangle$ between the carriers $X$ and $Y$ of $\langle X, k\rangle$ and $\langle Y, \ell\rangle$ lifts to a coalgebraic ordered bisimulation if there exists a coalgebra structure $\widetilde{R}: R \rightarrow B R$ making the first leg $r_{1}$ a lax coalgebra morphism and the second leg $r_{2}$ a (strict) coalgebra morphism:

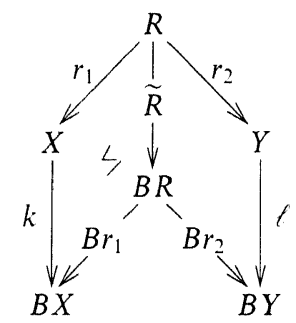

In an order-enriched category, the internal inequality IQ $(X)$ of an object $X$ can be defined by means of the following order-enriched version of the notion of pullbacks.

Definition 6.2. The ordered pullback of a pair $f: X \rightarrow Z$ and $g: Y \rightarrow Z$ of morphisms in a Poset-category having the same codomain is a span $\langle P, p: P \rightarrow X, q: P \rightarrow Y\rangle$ such that $f \circ p \leqslant g \circ q$ and for every span $\left\langle P^{\prime}, p^{\prime}: P^{\prime} \rightarrow X, q^{\prime}: P^{\prime} \rightarrow Y\right\rangle$ such that $f \circ p^{\prime} \leqslant g \circ q^{\prime}$ there exists a unique morphism $u: P^{\prime} \rightarrow P$ such that $p^{\prime}=p \circ u$ and $q^{\prime}=q \circ u$. Diagrammatically:

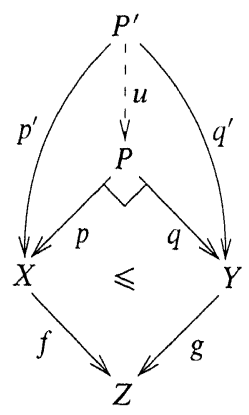

(Compare this with Diagram (6) in Section 4.)

(Ordered pullbacks are instances of the 'comma-objects' of Kelly (1989, Section 4).)

In Poset (and $\underline{\text { Pppo }}_{\perp}$ ), the ordered pullback of two morphisms $f: X \rightarrow Z$ and $g: Y \rightarrow Z$ is given by the set

$$
\{<x, y>\in X \times Y \mid f(x) \leqslant g(y)\}
$$


ordered componentwise $\left.\left.(<x, y\rangle \leqslant<x^{\prime}, y^{\prime}\right\rangle \Longleftrightarrow x \leqslant x^{\prime}, y \leqslant y^{\prime}\right)$. Thus, in particular, the internal inequality of a poset (or cppo) $X$

$$
\mathrm{IQ}(X)=\left\{<x, x^{\prime}>\in X \times X \mid x \leqslant x^{\prime}\right\}
$$

is the ordered kernel pair of the identity on $X$ :

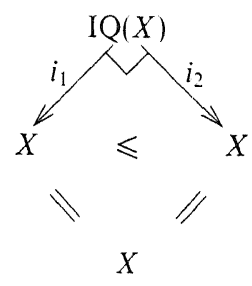

Proposition 6.2. (Fiore 1996b) For every coalgebra, the internal inequality lifts to a coalgebraic ordered bisimulation.

Proof: The proof is very much the same as that for Proposition 4.2; the coalgebra structure for $\mathrm{IQ}(X)$ is the one obtained by replacing $e_{1}=e_{2}: \operatorname{EQ}(X) \rightarrow X$ with $i_{2}: \mathrm{IQ}(X) \rightarrow X$. Notice that the fact that $i_{1}$ needs only to be lax with respect to the resulting coalgebra allows for the proposition to hold.

Proposition 6.3. The internal inequality of the maximally-final coalgebra lifts to the maximally-final ordered bisimulation.

Proof. Let us adapt the proof of Proposition 4.3. First, replace bisimulations by ordered bisimulations and take $f$ and $g$ in (7) to be lax coalgebra morphisms. Second, notice that replacing $\operatorname{EQ}(\widehat{B})$ by $\operatorname{IQ}(\widehat{B})$ in $(8)$, one still has, by the universal property of ordered pullbacks, a unique mediating morphism $u: R \rightarrow \operatorname{IQ}(\widehat{B})$ from $R$ to the internal inequality of the final coalgebra $\operatorname{IQ}(\widehat{B})$; this shows that $\operatorname{IQ}(\widehat{B})$ is final in the category of coalgebraic ordered bisimulations. It remains to prove maximality. For any other pair of lax morphisms $f$ and $g$ from $\langle X, k\rangle$ and $\langle Y, \ell\rangle$ to the final coalgebra, one has a mediating morphism $v: R \rightarrow \operatorname{IQ}(\widehat{B})$ and, by Proposition 6.1, that $f \leqslant k^{(\mathrm{a}}$ and $g \leqslant \ell^{\mathrm{a}}$. Then

$$
\left.\begin{array}{l}
i_{1} \circ v=f \circ r_{1} \leqslant k^{a} \circ r_{1}=i_{1} \circ u \\
i_{2} \circ v=g \circ r_{2} \leqslant \ell^{\mathrm{a}} \circ r_{2}=i_{2} \circ u
\end{array}\right\} \Rightarrow v \leqslant u,
$$

because, as one can check, the two legs of the inequality are jointly order monic.

\subsection{Order-enriched final coalgebra semantics}

Following Section 4.2, if ordered pullbacks lift to ordered bisimulations of an endofunctor $B$ on $\underline{C p p o}_{\perp}$, then the final ordered bisimulation between two $B$-coalgebras $\langle X, k\rangle$ and $\langle Y, \ell\rangle$ exists, and it is the ordered pullback of the coinductive extensions $k^{\mathrm{a}}$ and $\ell^{\mathrm{a}}$. Let us write ' $\lesssim$ ' for this ordered bisimulation (omitting the superscript $k, f$ ). Then, for every $x \in X$ and $y \in Y$,

$$
x \lesssim y \Longleftrightarrow k^{\varrho}(x) \leqslant \ell^{a}(y) .
$$


(The implication from left to right follows from Proposition 6.3, while the converse follows from the assumption that ordered pullbacks lift to ordered $B$-bisimulations.) Semantically, we have the following theorem.

Theorem 6.2. (Ordered full abstraction) Let $\mathbb{I}-\mathbb{\rrbracket}:$ Prog $\rightarrow B(\operatorname{Prog})$ be a coalgebraic operational model of a language with programs $P \in$ Prog and behaviour $B:$ Cppo $\rightarrow$ $\underline{\mathrm{Cppo}}_{\perp}$ such that ordered pullbacks lift to ordered $B$-bisimulations. Then for all $P, P^{\prime} \in$ Prog,

$$
P \lesssim P^{\prime} \Longleftrightarrow \llbracket P \rrbracket^{@} \leqslant \llbracket P^{\prime} \rrbracket^{@}
$$

\subsection{An example: deterministic transition systems with divergence}

We will give an example of fully abstract final coalgebra semantics in this order-enriched setting.

Consider the endofunctor

$$
B X=1_{\perp}+\left(A c t_{\perp} \otimes X_{\perp}\right)
$$

on $\underline{C p p o}_{\perp}$, where the endofunctor $X \mapsto X_{\perp}$ is the lifting functor adding a new least element to a cppo, the functor ' $\otimes$ ' is the smash product of cppos (obtained from the cartesian product by identifying all pairs containing a bottom), and the functor ${ }^{\prime}+$ ' is the categorical coproduct of $\mathrm{Cppo}_{\perp}$.

The final coalgebra of the above endofunctor $B$ is the set of finite, infinite, and partial words, the latter being finite words ending with $\perp$, such as, for example, $a b a \perp$. There is no relation between complete words, but any word $w$ is greater than a partial word $w^{\prime} \perp$ if $w$ extends $w^{\prime}$ (that is, $w=w^{\prime} w^{\prime \prime}$ for some non-empty word $w^{\prime \prime}$ ); for example, $a b \perp<a b a \perp<a b a a$. In particular, if Act is a singleton, the final coalgebra of the above behaviour is given by the 'lazy' or 'oblique natural numbers' - cf. Freyd (1990).

Next, consider deterministic transition systems in which a state, besides performing an action or being inert, can diverge. These can be modelled as coalgebras of the above endofunctor as follows. Regard the set $X$ of states of the transition system as a flat cppo (thus possibly adjoining a least element $\perp_{X}$ ). Notice that, for every cppo $X$, in Cpo (or $\underline{\text { Set) }}$ one can prove the isomorphism

$$
B(X)=1_{\perp}+A c t_{\perp} \otimes X_{\perp} \cong(1+A c t \times X)_{\perp},
$$

where the product and coproduct inside the parentheses are in Cpo rather than in Cppo $_{\perp}$. Let us denote the least element of $B X$ as $\perp_{\text {new }}$. One can then model deterministic transition systems with divergence as strict functions $k: X \rightarrow(1+\text { Act } \times X)_{\perp}$ by putting $k\left(\perp_{X}\right)=\perp_{\text {new }}$ and, for every other $x$ in $X$,

$$
k(x)= \begin{cases}\perp_{\text {new }} & \text { if } x \text { diverges } \\ * & \text { if } x \text { is inert } \\ \left.<a, x^{\prime}\right\rangle & \text { if } x \stackrel{a}{\longrightarrow} x^{\prime} .\end{cases}
$$

An ordered bisimulation $R$ between the carriers $X$ and $Y$ of two such transition systems satisfies, for all $x R y$, 
1 if $x$ is inert (that is, $k(x)=*$ ), then $y$ is inert also, and

2 if $x \stackrel{a}{\longrightarrow} x^{\prime}$ (that is, $k(x)=<a, x^{\prime}>$ ), then $y \stackrel{a}{\longrightarrow} y^{\prime}$ and $x^{\prime} R y^{\prime}$

3 if $x$ does not diverge (that is, $k(x) \neq \perp_{\text {new }}$ ), then

(a) if $y$ is inert, then $x$ is inert also, and

(b) if $y \stackrel{a}{\longrightarrow} y^{\prime}$, then $x \stackrel{a}{\longrightarrow} x^{\prime}$ and $x^{\prime} R y^{\prime}$

4 if $x$ diverges (that is, $k(x)=\perp$ ) and $y \stackrel{a}{\longrightarrow} y^{\prime}$, then $x^{\prime} R y^{\prime}$ for some $x^{\prime}$ in $X$.

Conversely, every span $R$ between the carriers $X$ and $Y$ of two (coalgebras modelling) deterministic transition systems with divergence that satisfies Properties 1-4 above is an ordered bisimulation for the behaviour endofunctor. These are known, in the nondeterministic setting, as partial bisimulations (Abramsky 1991).

An example of an ordered $B$-bisimulation is the ordered pullback (in Cppo ) of two coalgebra homomorphisms $f:\langle X, k\rangle \rightarrow\langle Z, h\rangle$ and $g:\langle Y, \ell\rangle \rightarrow\langle Z, h\rangle$. Indeed, a $B$ coalgebra structure lifting the ordered pullback $R$ to an ordered $B$-bisimulation can be defined by putting, for every pair $\langle x, y\rangle$ in $R$,

$$
<x, y>\mapsto \begin{cases}* & \text { if } k(x)=\perp_{\text {new }}, \ell(y)=* \\ <a, \perp_{X}, y^{\prime}> & \text { if } k(x)=\perp_{\text {new }}, \ell(y)=<a, y^{\prime}> \\ \perp_{\text {new }} & \text { if } k(x)=\perp_{\text {new }}=\ell(y) \\ * & \text { if } k(x)=*=\ell(y) \\ <a, x^{\prime}, y^{\prime}> & \text { if } k(x)=<a, x^{\prime}>, \ell(y)=<a, y^{\prime}>.\end{cases}
$$

Notice that these are all the case distinctions that can occur. For instance, $k(x) \neq \perp_{\text {new }}=$ $\ell(y)$ is not possible because

$$
(1+A c t \times f)_{\perp}(k(x))=h(f(x)) \leqslant h(g(y))=(1+A c t \times g)_{\perp}(\ell(y)) .
$$

In particular, one can take $f$ and $g$ to be the coinductive extensions of $k$ and $\ell$, respectively. Therefore, by Theorem 6.2, the final coalgebra semantics of deterministic programs with divergence is fully abstract with respect to partial bisimulation.

\section{Metric spaces}

One of the advantages of working in an order-enriched setting is that one can verify not only the equality between computations, but also when a computation is better than another in the sense that it contains more information. For instance, under the standard prefix ordering on words, a computation represented by the $a_{1} \cdots a_{n} b$ is better than one represented by the word $a_{1} \cdots a_{n}$. Still, in the order-enriched setting one is not able to compare computations that share a common prefix but then differ, such as, for example, $a_{1} \cdots a_{n} b$ and $a_{1} \cdots a_{n} c$. In order to do this, one can introduce a natural notion of distance between computations.

The intuition is that the more two computations (programs) are (behave) the same, the smaller their distance should be. In the deterministic setting, with behaviours of type $B X=1+A c t \times X(c f$. Section 2.2), one can say that the distance between two words is $2^{-n}$, for $n$ the length of the longest common prefix between the two words. This leads to the use of (ultra) metric spaces in semantics. 
A metric space is a set $X$ together with a distance function $d_{X}: X \times X \rightarrow[0, \infty]$ mapping each two elements of $X$ to a non-negative real number. (It is convenient to include $\infty$ in the range of this distance function, even though one can always down-scale distances to the interval $[0,1]$.) Furthermore, a distance has to satisfy the following three axioms:

$1 \quad d_{X}(x, y)=0 \Longleftrightarrow x=y$

$2 \quad d_{X}(x, z) \leqslant d_{X}(x, y)+d_{X}(y, z)$

$3 \quad d_{X}(x, y)=d_{X}(y, x)$.

The second axiom is called the triangular inequality. When a stronger form of triangular inequality holds, namely $d_{X}(x, z) \leqslant \max \left\{d_{X}(x, y), d_{X}(y, z)\right\}$, then $\left\langle X, d_{X}\right\rangle$ is an ultra-metric space. In semantics one usually works with these more specific structures, but it is convenient to state the general theorems in the full generality of metric spaces.

In metric spaces, the available mathematical tool most relevant for semantics is Banach's Theorem. This theorem ensures the existence of a unique fixed point for a large class of endofunctions on complete metric spaces, that is, on metric spaces closed under limits of 'Cauchy sequences'. The endofunctions at stake are the contractive ones, that is, functions $f: X \rightarrow X$ such that, for some $0 \leqslant \epsilon<1$,

$$
d\left(f(x), f\left(x^{\prime}\right)\right) \leqslant \epsilon \cdot d\left(x, x^{\prime}\right)
$$

for all $x, x^{\prime} \in X$. (Here the subscript $X$ is omitted from $d_{X}$; the same is done in the rest of this paper whenever possible.)

Theorem 7.1. (Banach) Every contractive endofunction $f$ on a complete metric space has a unique fixed point $f x(f)=f(f i x(f))$.

Banach's Theorem carries over to the higher level of categories and functors as follows. Consider the category Metric with objects metric spaces and with morphisms the nonexpansive functions between them, that is, the functions $f: X \rightarrow Y$ such that

$$
d_{Y}\left(f(x), f\left(x^{\prime}\right)\right) \leqslant d_{X}\left(x, x^{\prime}\right)
$$

for all $x, x^{\prime} \in X$.

Observation 7.1. The set $\operatorname{Metric}(X, Y)$ of non-expansive functions between two metric spaces is itself a metric space, with distance

$$
d(f, g)=\sup _{x \in X}\left\{d_{Y}(f(x), g(x))\right\}
$$

for all non-expansive functions $f, g: X \rightarrow Y$. In other words, the hom-sets of Metric are objects of Metric itself. Similarly, one has that in the subcategory $\underline{\mathrm{Cms}}$ of complete metric spaces and non-expansive functions, the hom-sets are themselves complete metric spaces, with (16) as distance.

Therefore, one can define an endofunctor $F$ on Metric (or on the subcategory $\underline{\mathrm{Cms}}$ ) to be locally contractive if, for some $0 \leqslant \epsilon<1$,

$$
d(F(f), F(g)) \leqslant \epsilon \cdot d(f, g)
$$

for all metric spaces $X, Y$, and for all pairs of parallel non-expansive functions $f, g: X \rightarrow$ $Y$ between them. 
Theorem 7.2. Every locally contractive endofunctor $F$ on the category of complete metric spaces and non-expansive functions has a unique fixed point $F i x(F) \cong F(F i x(F))$.

Proof. The proof is based on America and Rutten (1989), where a category $\underline{\mathrm{Cms}}^{E}$ of complete metric spaces is considered that has as morphisms (a metric version of Smyth and Plotkin's) 'embedding-projection' pairs - $c f$. Section 6.1. Then a notion of 'contractivity' for endofunctors on that category is defined, and it is shown that contractive functors have a unique fixed point. The present theorem follows from the observation (due to Gordon Plotkin) that every locally contractive functor on $\mathrm{Cms}$ induces a contractive functor on $\underline{\mathrm{Cms}}^{E}$. (For details see Rutten and Turi (1993).)

As shown by the following proposition, the above theorem gives a foundation for both induction and coinduction.

Proposition 7.1. Every fixed point $F i x(F) \cong F(F i x(F))$ of a locally contractive functor $F: \underline{\mathrm{Cms}} \rightarrow \underline{\mathrm{Cms}}$ is both an initial algebra and a final coalgebra for $F$.

Proof. Let $\varphi: F i x(F) \cong F(F i x(F))$ be the $F$-coalgebra structure given by the fixed point isomorphism and let $\langle X, k\rangle$ be an $F$-coalgebra. Define $\Phi: \underline{\mathrm{Cms}}(X, F i x(F)) \rightarrow$ $\underline{\mathrm{Cms}}(X, F i x(F))$ by, for all $f$,

$$
\Phi(f)=\varphi^{-1} \circ F(f) \circ k
$$

Suppose $F$ is locally contractive with factor $\epsilon$. Then $\Phi$ is a contraction with factor $\epsilon$ : for all $f_{1}, f_{2} \in \underline{\mathrm{Cms}}(X, F i x(F))$,

$$
\begin{aligned}
d\left(\Phi\left(f_{1}\right), \Phi\left(f_{2}\right)\right) & =\sup _{x \in X}\left\{d_{\operatorname{Fix}(F)}\left(\Phi\left(f_{1}\right)(x), \Phi\left(f_{2}\right)(x)\right)\right\} \\
& =\sup _{x \in X}\left\{d_{\operatorname{Fix}(F)}\left(\left(\varphi^{-1} \circ F\left(f_{1}\right) \circ k\right)(x),\left(\varphi^{-1} \circ F\left(f_{2}\right) \circ k\right)(x)\right)\right\} \\
& \leqslant \sup _{y \in F(X)}\left\{d_{\operatorname{Fix}(F)}\left(\left(\varphi^{-1} \circ F\left(f_{1}\right)\right)(y),\left(\varphi^{-1} \circ F\left(f_{2}\right)\right)(y)\right)\right\} \\
& \leqslant \sup _{y \in F(X)}\left\{d_{\operatorname{Fix}(F)}\left(F\left(f_{1}\right)(y), F\left(f_{2}\right)(y)\right)\right\}\left(\varphi^{-1} \text { is non-expansive }\right) \\
& =d\left(F\left(f_{1}\right), F\left(f_{2}\right)\right) \\
& \leqslant \epsilon \cdot d\left(f_{1}, f_{2}\right)(F \text { is locally contractive }) .
\end{aligned}
$$

By Banach's Theorem, $\Phi$ has a unique fixed point $f i x(\Phi): X \rightarrow F i x(F)$. Moreover

$$
\varphi \circ f i x(\Phi)=\varphi \circ \Phi(f x(\Phi))=\varphi \circ \varphi^{-1} \circ F(f i x(\Phi)) \circ k=F(\text { fix }(\Phi)) \circ k,
$$

which shows that $f i x(\Phi)$ is a coalgebra morphism from $\langle X, k\rangle$ to $\langle F i x(F), \varphi\rangle$. Since any such coalgebra morphism is also a fixed point of $\Phi$, fix $(\Phi)$ is also the unique coalgebra morphism, which shows that $\langle F i x(F), \varphi\rangle$ is a final $F$-coalgebra. A dual argument shows that $\left\langle F i x(F), \varphi^{-1}\right\rangle$ is an initial $F$-algebra.

The proof of the above proposition does not require that the metric space $X$ be complete, because for the hom-set $\operatorname{Metric}(X, Y)$ to be complete it is sufficient that $Y$ be complete. Therefore, we have the following corollary.

Corollary 7.1. A final coalgebra (initial algebra) of a locally contractive endofunctor $F$ on $\underline{\mathrm{Cms}}$ that extends to an endofunctor $F^{\prime}$ on Metric is also a final $F^{\prime}$-coalgebra (initial $F^{\prime}$-algebra) in Metric. 
As a consequence, one can consider metric operational models that are not complete and still have a final coalgebra semantics. Similarly, bisimulations in the metric setting need not be complete objects.

\subsection{Metric-enriched categories}

The categories Metric and $\underline{\mathrm{Cms}}$ are both self-enriched categories in the sense of Section 6.1. The corresponding notion of a Metric-functor, however, is not local contractivity, but the milder one of 'locally non-expansiveness', which comprehends all functors used in metric semantics. However, every locally non-expansive endofunctor can be turned into a locally contractive one by composing it with a trivial 'shrinking' endofunctor.

The categorical product of two metric spaces $\left\langle X, d_{X}\right\rangle$ and $\left\langle Y, d_{Y}\right\rangle$ is the cartesian product of their carriers equipped with the distance

$$
d\left(<x, x^{\prime}>,<y, y^{\prime}>\right)=\max \left\{d_{X}\left(x, x^{\prime}\right), d_{Y}\left(y, y^{\prime}\right)\right\} .
$$

However, the composition of non-expansive functions is not non-expansive with respect to the categorical product of hom-sets. Therefore, a different notion of distance is needed, namely

$$
d\left(<x, x^{\prime}>,<y, y^{\prime}>\right)=d_{X}\left(x, x^{\prime}\right)+d_{Y}\left(y, y^{\prime}\right) .
$$

Equipped with this distance, the cartesian product $X \times Y$ of the carriers is called the tensor product $\left\langle X, d_{X}\right\rangle \otimes\left\langle Y, d_{Y}\right\rangle$ of two metric spaces. (This tensor product is associative and commutative, and it has the singleton metric space 1 as a unit, making of $\underline{\mathrm{Cms}}$ a '(symmetric) monoidal category' in the sense of Mac Lane (1971, Section VII.1).) One can check that the composition of non-expansive functions, viewed, for all metric spaces $X, Y, Z$, as a function

$$
\circ: \underline{\operatorname{Metric}}(Y, Z) \otimes \underline{\operatorname{Metric}}(X, Y) \rightarrow \underline{\operatorname{Metric}}(X, Z)
$$

is non-expansive. The same holds for its restriction to $\mathrm{Cms}$. Therefore, because of $\mathrm{Ob}$ servation 7.1, the categories Metric and $\underline{\mathrm{Cms}}$ are Metric-enriched categories and $\underline{\mathrm{Cms}}$ is Cms-enriched. A Metric-endofunctor $F$ on a Metric-category is then an endofunctor such that

$$
d(F(f), F(g)) \leqslant d(f, g)
$$

for all metric spaces $X, Y$, and for all pairs of parallel non-expansive functions $f, g: X \rightarrow$ $Y$ between them. In other words, the Metric-endofunctors are the 'locally non-expansive' ones.

Definition 7.1. (Down-scalers and shrunk functors) The down-scaler endofunctor $I d_{\epsilon}$ : Metric $\rightarrow$ Metric, with scaling factor $0 \leqslant \varepsilon<1$, is the functor that 'shrinks' the distance $d$ of a metric space by mapping it to $\epsilon \cdot d$, while on carriers and on morphisms it behaves as the identity:

$$
I d_{\epsilon}\langle X, d\rangle=\langle X, \epsilon \cdot d\rangle .
$$

A shrunk endofunctor $F:$ Metric $\rightarrow$ Metric is an endofunctor that can be decomposed either as $F^{\prime} \circ I d_{\epsilon}$ or as $I d_{\epsilon} \circ F^{\prime}$, for some endofunctor $F^{\prime}$ and some $0 \leqslant \epsilon<1$. 
Clearly, every shrunk locally non-expansive endofunctor on Metric (and on $\underline{\mathrm{Cms}}$ ) is locally contractive, therefore, by Theorem 7.2 and Proposition 7.1, the following theorem holds.

Theorem 7.3. Every shrunk Metric-endofunctor on $\underline{\mathrm{Cms}}$ has both an initial algebra and a final coalgebra and, moreover, they are canonically isomorphic. (Compare this with Theorem 6.1.)

For example, consider the behaviour endofunctor $B X=1+$ Act $\times X$ on Set. It extends to a Metric-endofunctor on $\mathrm{Cms}$ by imposing the discrete bounded ultrametric on Act (that is, $d\left(a, a^{\prime}\right)=0$ if $a=a^{\prime}$ and $d\left(a, a^{\prime}\right)=1$ if $\left.a \neq a^{\prime}\right)$. Notice that products and coproducts of spaces with discrete metric are like in Set. By precomposing this behaviour with the (typical) down-scaler $I d_{\frac{1}{2}}$, one obtains the shrunk Cms-endofunctor $B\langle X, d\rangle=1+A c t \times\left\langle X, \frac{1}{2} d\right\rangle$. One can check that the carrier of its final coalgebra and initial algebra given by the above theorem is the set $A c t^{\infty}=A c t^{*} \cup A c t^{*}$ of finite and infinite words over the alphabet Act. Its distance is the one described at the beginning of this section on metric spaces, namely two words have distance $2^{-n}$ if $n$ is the length of the longest common prefix between them.

\subsection{Reconciling partial orders with metric spaces}

Structures that combine the qualitative (asymmetric) information given by partial orders with the quantitative information given by (symmetric) metric spaces are the quasimetric spaces, that is, metric spaces in which the (third) axiom stating the symmetry of the distance functions is omitted. Thus a quasimetric space is a set $X$ equipped with a function $d: X \times X \rightarrow[0, \infty]$ such that, for all $x, y$, and $z$ in $X$,

$1 d(x, y)=0$ and $d(y, x)=0 \Longleftrightarrow x=y$

$2 d(x, z) \leqslant d(x, y)+d(y, z)$.

Clearly, the category QMetric of quasimetric spaces and non-expansive functions contains Metric as a full subcategory. Moreover, thanks to the asymmetry of the distance, the category Poset of partial orders and monotone functions fully and faithfully embeds in QMetric. This embedding maps a partial order $\leqslant$ on a set $X$ to the following distance $d_{\leqslant}$ on $X$ :

$$
d_{\leqslant}(x, y)= \begin{cases}0 & \text { if } x \leqslant y \\ \infty & \text { otherwise }\end{cases}
$$

Monotone functions between partial orders are non-expansive functions with respect to their corresponding quasi-metrics.

A crucial contribution to the theory of quasimetric spaces has been the introduction in Smyth (1988) of notions of limit and completeness for quasimetric spaces generalizing both the notions of least upper bound of an $\omega$-chain and of (ordinary) metric limit of a Cauchy sequence. The corresponding category Cqms of complete quasimetric spaces has as morphisms the non-expansive functions that are continuous in the sense that they preserve Cauchy sequences and the corresponding limits. Notice that for metric spaces, non-expansive functions are always continuous, while for quasimetric spaces the two notions are incomparable - see Rutten (1996, Remark 3.6). Also, contractive functions are 
not necessarily continuous, and Banach's Theorem holds for complete quasimetric spaces only under the additional assumption that the endofunction at stake is continuous.

As with Cms, Banach's Theorem carries over to the level of Cqms (Rutten 1996, Theorem 7.3). This allows for the following generalization of Theorem 7.3. First notice that the category Cqms is, like $\mathrm{Cms}$, self-enriched (with respect to the same tensor product as for ordinary metric spaces), thus a Cqms-endofunctor on Cqms is an endofunctor which is both locally non-expansive and locally continuous. Thus we have the following theorem.

Theorem 7.4. Every shrunk Cqms-endofunctor on Cqms has both an initial algebra and a final coalgebra and, moreover, they are canonically isomorphic.

Proof. See Rutten (1996, Theorem 7.3).

It is an open question whether by, on the one hand, removing the shrunkness condition and by, on the other hand, working in the category of pointed complete quasi-metric spaces and strict continuous and non-expansive functions, one would still have the same result, thus generalizing Theorem 6.1 .

Generalized Metric Spaces. Smyth's notion of Cauchy sequence for quasimetric spaces has been generalized both in Flagg and Kopperman (1997) and in K. R. Wagner's thesis, Wagner (1994). Wagner's notion of limit is made parametric in a quantale $\Omega$ : for $\Omega$ equal to the two-elements lattice $\perp \leqslant T$, it specializes to the standard notion of an $\omega$-chain in a partial order; for $\Omega$ equal to $[0, \infty]$ it specializes to Smyth's Cauchy sequence. Wagner uses the parameter $\Omega$ to generalize the standard constructions of initial algebras (alias final coalgebras) for $\underline{\mathrm{Cppo}}_{\perp}$ and $\underline{\mathrm{Cms}}$.

Wagner's work is based on a seminal article by Lawvere (Lawvere 1973), where metric spaces, partial orders, and categories are all shown to be instances of the notion, described in Section 6.1, of a $\underline{V}$-category, for different (monoidal) categories $\underline{\mathrm{V}}$.

Metric spaces are obtained by putting $\underline{V}=[0, \infty]$. The morphisms of $[0, \infty]$ are given by its reverse order, that is, $r \rightarrow r^{\prime}$ if and only if $r \geqslant r^{\prime}$; the tensor product is the sum of reals. Then, a $[0, \infty]$-category is a generalized metric space, that is, a set $X$ equipped with a function $d: X \times X \rightarrow[0, \infty]$ such that, for all $x, y$, and $z$ in $X$,

$1^{\prime} d(x, x)=0$

$2 d(x, z) \leqslant d(x, y)+d(y, z)$.

Notice that two elements with distance 0 need not be equal. Clearly, every metric space is also a generalized metric space. Interestingly, if the 'max' operator rather than the sum between reals is taken as tensor of $[0, \infty]$, one has that a $[0, \infty]$-category is a generalized ultrametric space, with the max operator replacing the sum in the triangular inequality axiom.

Partial orders are obtained by putting $\underline{\mathrm{V}}=\underline{2}$, the category with two elements $\perp$ and $T$ and only one non-identity morphism, namely $\perp \leqslant T$. With the logical 'and' as the tensor product of 2 , a 2 -category is then nothing but a pre-order.

Next, the notion of a $\underline{V}$-functor specializes to that of a monotone function for $\underline{V}=\underline{2}$, and to that of a non-expansive function for $\underline{V}=[0, \infty]$. Thus, at a higher level, a Posetfunctor can be seen as a locally 2 -functor and, similarly, a Metric-functor can be seen as a locally $[0, \infty]$-functor. 
The conceptual advantage of regarding (generalized) metric spaces as $[0, \infty]$-categories goes well beyond providing a unifying framework. For instance, in $\underline{V}$-category one has the notion of a bimodule and, as argued in Lawvere (1973), one can regard bimodules as being ' $V$-valued relations'. In particular, a bimodule $R$ between two metric spaces $X$ and $Y$ (regarded as $[0, \infty]$-categories) is a function

$$
R: X \times Y \rightarrow[0, \infty]
$$

satisfying suitable conditions Lawvere (1973, page 159). The value of $R$ at a pair $(x, y)$ is the 'truth value of the $R$-relatedness of $x$ to $y$ '. With such a notion of $[0, \infty]$-valued relations, one can then define a new notion of metric bisimulation as follows. Consider, for simplicity, the deterministic transition systems given by the coalgebras in Metric of the behaviour

$$
B=1+\left(A c t \times I d_{\frac{1}{2}}\right): \text { Metric } \rightarrow \text { Metric. }
$$

Then a $[0, \infty]$-valued relation $R$ on the states of one such deterministic transition system $k: X \rightarrow B X$ could be called a 'metric bisimulation' if it satisfies

$$
x R y= \begin{cases}0 & \text { if } k(x)=*=k(y) \\ \frac{1}{2} \cdot \epsilon & \text { if } k(x)=<a, x^{\prime}>, k(y)=<a, y^{\prime}>, x^{\prime} R y^{\prime}=\epsilon \\ 1 & \text { otherwise. }\end{cases}
$$

The idea is that $x R y=2^{-n}$ if for $n$ transition steps, $x$ and $y$ behave the same and then differ. In particular, $x R y=0$ if $x$ and $y$ are bisimilar in the ordinary sense.

A coalgebraic generalization of the above notion of a metric bisimulation is outside the scope of the present work.

\section{References}

Abramsky, S. (1990) The lazy lambda calculus. In: Turner, D. (ed.) Research Topics in Functional Programming, Addison-Wesley 65-116.

Abramsky, S. (1991) A domain equation for bisimulation. Information and Computation 92 161-218.

Aczel, P. (1988) Non-well-founded sets, CSLI Lecture Notes 14.

Aczel, P. (1994) Final universes of processes. In: Mathematical Foundations of Programming Semantics, Proc. 9th Int. Conf., New Orleans, LA, USA, April 1993. Springer-Verlag Lecture Notes in Computer Science 802 1-28.

Aczel, P. and Mendler, N. (1989) A final coalgebra theorem. In: Pitt, D. et al. (eds.) Proc. category theory and computer science. Springer-Verlag Lecture Notes in Computer Science 389 357-365.

America, P. and Rutten, J. (1989) Solving reflexive domain equations in a category of complete metric spaces. Journal of Computer and System Sciences 39 (3) 343-375.

Arbib, M. and Manes, E. (1980) The greatest fixpoint approach to data types. In: Proc. of the 3rd workshop meeting on Categorical and Algebraic Methods in Computer Science and System Theory. See also Arbib and Manes (1982).

Arbib, M. and Manes, E. (1982) Parametrized data types do not need highly constrained parameters. Information and Control 52 139-158.

Arnold, A. and Dicky, A. (1989) An algebraic characterization of transition system equivalences. Information and Computation 82 198-229. 
Baldamus, M. (1994) A non-well-founded sets semantics for observation congruence over full CCS. Tech. rep. Berlin University of Technology.

Barr, M. (1993) Terminal coalgebras in well-founded set theory. Theoretical Computer Science 144 (2) 299-315.

Barwise, J. and Etchemendy, J. (1987) The Liar: An Essay in Truth and Circularity, Oxford University Press.

Bonsangue, M., Breugel, F.V. and Rutten, J. (1998) Generalized ultrametric spaces: completion, topology, and powerdomains via the Yoneda embedding. Theoretical Computer Science 193 1-51.

Casley, R., Crew, R., Meseguer, J. and Pratt, V. (1991) Temporal structures. Math. Structures in Comp. Sci. 1 (2) 179-213.

de Bakker, J. and de Vink, E. (1996) Control Flow Semantics, The MIT Press.

de Bakker, J. and Rutten, J. (eds.) (1992) Ten Years of Concurrency Semantics, World Scientific, Selected papers of the Amsterdam Concurrency Group.

Fiore, M. (1996a) Axiomatic Domain Theory in Categories of Partial Maps, Distinguished Dissertations Series, Cambridge University Press.

Fiore, M. (1996b) A coinduction principle for recursive data types based on bisimulation. Information and Computation 127 (2) 186-198.

Fiore, M. and Plotkin, G. (1992) On compactness and Cpo-enriched categories. In: Winskel, G. (ed.) Proceedings of the CLICS Workshop (23-27 March 1992). DAIMI PB 397-II Computer Science Department, Aarhus University 571-584.

Fiore, M. and Plotkin, G. (1994) An axiomatisation of computationally adequate domain-theoretic models of FPC. $9^{\text {th }}$ LICS Conf., IEEE, Computer Society Press 92-102.

Flagg, B. and Kopperman, R. (1997) Continuity spaces: Reconciling domains and metric spaces. Theoretical Computer Science 177 (1) 111-138.

Forti, M. and Honsell, F. (1983) Set theory with free construction principles. Annali Scuola Normale Superiore, Pisa X (3) 493-522.

Freyd, P. (1990) Recursive types reduced to inductive types. Proc. Fifth IEEE Symp. on Logic In Computer Science, IEEE Computer Society Press 498-507.

Freyd, P. (1991) Algebraically complete categories. In: Carboni, A., Pedicchio, M. and Rosolini, G. (eds.) Category Theory - Proc. of the Int'l Conf. held in Como, Italy, July 1990. Springer-Verlag Lecture Notes in Mathematics 1488 95-104.

Freyd, P. (1992) Remarks on algebraically compact categories. In: Fourman, M., Johnstone, P. and Pitts, A. (eds.) Applications of Category Theory in computer science. London Mathematical Society Lecture Notes Series, Cambridge University Press 177 95-106.

Goguen, J., Thatcher, J. and Wagner, E. (1978) An initial algebra approach to the specification, correctness and implementation of abstract data types. In: Yeh, R. (ed.) Current Trends in Programming Methodology, Prentice Hall IV 80-149.

Hartonas, C. (1997) Semantics of finite delay. Theoretical Computer Science 176 (1-2) 205-234.

Hermida, C. and Jacobs, B. (1995) Induction and coinduction via subset types and quotient types. In: Informal Proceedings of the Joint CLICS-TYPES Workshop on Categories and Type Theory. Prog. Meth. Group, Report 85, Göteborg Univ. and Chalmers Univ. of Techn.

Honsell, F. and Lenisa, M. (1995) Final semantics for untyped $\lambda$-calculus. In: Dezani-Ciancaglini, M. and Plotkin, G. (eds.) Typed Lambda calculi and applications: second international conference. Springer-Verlag Lecture Notes in Computer Science $\mathbf{9 0 2}$.

Joyal, A. and Moerdijk, I. (1995) Algebraic Set Theory, London Mathematical Society Lecture Note Series 220, Cambridge University Press.

Joyal, A., Nielsen, M. and Winskel, G. (1993) Bisimulation and open maps. In: Proc. Eighth IEEE Symp. on Logic In Computer Science 418-427. 
Kelly, M. (1982) Basic Concepts of Enriched Category Theory, Cambridge University Press.

Kelly, M. (1989) Elementary observations on 2-categorical limits. Bull. Aust. Math. Soc. 39 (2) 301-317.

Lawvere, F.W. (1973) Metric spaces, generalized logic, and closed categories. Rendiconti del Seminario Matematico e Fisico di Milano 43 135-166.

Lawvere, F. W. (1976) Variable quantities and variable structure in topoi. In: Heller, A. and Tierney, M. (eds.) Algebra, Topology, and Category Theory, Academic Press 101-131.

Levy, A. (1979) Basic Set Theory, Perspectives in Mathematical Logic, Springer-Verlag.

Mac Lane, S. (1971) Categories for the Working Mathematician, Graduate Texts in Mathematics 5, Springer-Verlag.

Mac Lane, S. (1986) Mathematics: Form and Function, Springer-Verlag.

Manes, E. (1976) Algebraic Theories, Graduate Texts in Mathematics 26, Springer-Verlag.

Milner, R. (1980) A Calculus of Communicating Systems. Springer-Verlag Lecture Notes in Computer Science 92.

Mukai, K. (1991) Constraint Logic Programming and the Unification of Information, Ph. D. thesis, Tokio Institute of Technology.

Park, D. (1981) Concurrency and automata on infinite sequences. In: Deussen, P. (ed.) Proceedings 5th GI Conference. Springer-Verlag Lecture Notes in Computer Science 104 167-183.

Paulson, L. C. (1995) A concrete final coalgebra theorem for ZF set theory. In: Dybjer, P. et al. (eds.) Types for Proofs and Programs '94. Springer-Verlag Lecture Notes in Computer Science 996 120-139.

Pitts, A. (1994a) A co-induction principle for recursively defined domains. Theoretical Computer Science 124 195-219.

Pitts, A. (1994b) Computational adequacy via 'mixed' inductive definitions. In: Mathematical Foundations of Programming Semantics, Proc. 9th Int. Conf., New Orleans, LA, USA, April 1993. Springer-Verlag Lecture Notes in Computer Science 802 72-82.

Plotkin, G. (1981) A structural approach to operational semantics. Technical Report DAIMI FN-19, Computer Science Department, Aarhus University.

Rutten, J. (1996) Elements of generalized ultrametric domain theory. Theoretical Computer Science 170 349-381.

Rutten, J. and Turi, D. (1993) On the foundations of final semantics: non-standard sets, metric spaces, partial orders. In: de Bakker, J. et al. (eds.) Proc. of the REX workshop Semantics Foundations and Applications. Springer-Verlag Lecture Notes in Computer Science 666 477-530.

Rutten, J. and Turi, D. (1994) Initial algebra and final coalgebra semantics for concurrency. In: de Bakker, J. et al. (eds.) Proc. of the REX workshop A Decade of Concurrency - Reflections and Perspectives. Springer-Verlag Lecture Notes in Computer Science 803 530-582.

Smyth, M. (1988) Quasi uniformities: reconciling domains with metric spaces. In: Main, M., Melton, A., Mislove, M. and Schmidt, D. (eds.) Proceedings of the 3rd Workshop on Mathematical Foundations of Programming Language Semantics. Springer-Verlag Lecture Notes in Computer Science 298 236-253.

Smyth, M. and Plotkin, G. (1982) The category-theoretic solution of recursive domain equations. SIAM J. Comput. 11 761-783.

Turi, D. (1996) Functorial Operational Semantics and its Denotational Dual. Ph. D. thesis, Free University, Amsterdam. (Available from <http://www.dcs.ed.ac.uk/home/dt>.)

Turi, D. (1997) Categorical modelling of structural operational rules: case studies. In: Moggi, E. and Rosolini, G. (eds.) Proc. $7^{\text {th }}$ CTCS Conference. Springer-Verlag Lecture Notes in Computer Science 1290 127-146. 
Turi, D. and Plotkin, G. (1997) Towards a mathematical operational semantics. Proc. $12^{\text {th }}$ LICS Conf., IEEE, Computer Society Press 280-291.

van Benthem, J. (1984) Correspondence theory. In: Gabbay, D. M. et al. (eds.) Handbook of Philosophical Logic II, Reidel 167-247.

Wagner, K. (1994) Solving recursive domain equations with enriched categories, Ph. D. thesis, Carnegie Mellon University, Pittsburgh. (Technical report CMU-CS-94-159.) 